\title{
Analysis of the heteroclinic connection in a singularly perturbed system arising from the study of crystalline grain boundaries
}

\author{
N. D. Alikakos ${ }^{\dagger}$ \\ Department of Mathematics, University of North Texas, \\ Denton, TX 76203-1430, USA \\ and University of Athens \\ P. C. FIFE \\ Department of Mathematics, University of Utah, \\ Salt Lake City, UT 84112, USA \\ G. FusCO ${ }^{\S}$ \\ Università degli Studi dell'Aquila, L'Aquila, Italy \\ AND \\ C. Sourdis ${ }^{\text {II }}$ \\ Mathematics Department, University of Athens, Athens, Greece
}

[Received 2 February 2005 and in revised form 29 November 2005]

\begin{abstract}
Mathematically, the problem considered here is that of heteroclinic connections for a system of two second order differential equations of Hamiltonian type, in which a small parameter $\epsilon$ conveys a singular perturbation. The motivation comes from a multi-order-parameter phase field model developed by Braun et al. [5] and [22] for the description of crystalline interphase boundaries. In this model, the smallness of $\epsilon$ is related to large anisotropy. The existence of such a heteroclinic, and its dependence on $\epsilon$, is proved. In addition, its robustness is investigated by establishing its spectral stability.
\end{abstract}

\section{Introduction}

\subsection{The model and prior results}

The physical context is that of crystals existing in several phases, and the general goal is to study the structure of interphase boundaries. The modeling framework uses order parameters as both microscopic and mesoscopic descriptors of the state of the material, with dynamics given by the gradient flow of a free energy functional involving the order parameters and their spatial gradients. Properties of the crystalline structure lead naturally to models based on several order parameters, which involve serious mathematical challenges not found in Cahn-Hilliard and AllenCahn equations and related single-order-parameter models. One new element is the set of invariants

\footnotetext{
$\dagger$ Supported by grants from the University of North Texas and the University of Athens. E-mail: nalikako@earthlink.net

‡E-mail: fife@math.utah.edu

§E-mail: fusco@mailhost.univaq.it

${ }^{\mathbb{I}}$ Supported by I.K.Y. E-mail: sour@dei.gr
} 
reflecting the crystallography of the material. This combined with the usual lack of convexity when there are phase transitions makes the problems rich but difficult. The geometry of the free energy function is generally quite intricate with many critical points, and the dependence of the surface energy on orientation is typically not convex.

The model we will be describing in this section, and which forms the basis of the analysis to follow, is that in [5] and [22]. It grew out of efforts to overcome the ad hoc approach which has been employed in single-order-parameter models to represent anisotropic interfaces. This model employs an energy functional which is intimately related to the crystalline lattice and is formulated in terms of physically based order parameters. The gradient energy terms are sums of squares of derivatives with coefficients which reflect the anisotropy. What results is a continuous (diffuse) description of an interface. One way to model anisotropic interfacial properties in a single-order-parameter diffuse interface theory is to allow the gradient energy coefficients and the mobility coefficient to depend on the spatial gradient of the order parameter, which is affected by the orientation of the interface. In this way the surface energy and the kinetic coefficient can be assigned a given anisotropy. While this approach allows a great deal of flexibility, it is also somewhat ad hoc. Another approach involves introducing anisotropy through generalized gradient energy terms that include higher order derivatives; this approach can also be difficult to justify on theoretical grounds.

On the other hand, the use of continuum models based on an underlying lattice such as we are considering, has the advantage that the anisotropy appears in a natural way, and correctly incorporates the crystal symmetries present.

Our description, in this section, of the crystalline framework and the resulting phase field model, will be brief; full details can be found in [5]. We begin with a textbook physical description of surface tension [19], since that concept is directly related to our construction of a free energy functional below. Surface tension can be thought of either as the force per unit length required to maintain an interface or as (in the present case) the energy per unit area necessary to generate an interface. On the microscopic level, surface tension can be related to the number of broken bonds, which is exactly what distinguishes atoms on the surface from those in the interior. Planar cuts of the crystal at different orientations have different numbers of broken bonds per unit of area, and this induces anisotropic surface tension.

In the present paper we will be dealing with 3D lattices, and with a type of crystal known as FCC (face-centered cubic). Quite analogous problems and methods hold for other structures; for example in the case of crystals of the hexagon-closely-packed (HCP) type the analogy is almost complete [16]. The FCC is a periodic arrangement of atoms whose unit cell is a cube with atoms occupying its corners and the centers of its faces. We easily count twelve closest neighbors to each atom. We can identify three distinguished planar cuts corresponding to the normal directions $\bar{n}=$ $(1 / \sqrt{3}, 1 / \sqrt{3}, 1 / \sqrt{3}), \bar{n}=(1,0,0), \bar{n}=(0,1 / \sqrt{2}, 1 / \sqrt{2})$ (Fig. 1). It is a simple exercise to count broken bonds for each of these orientations. We find respectively three, four, and five broken bonds per unit area.

Each unit cell in the FCC contains the equivalent of four whole atoms and so a tetrahedron can be associated with it (Fig. 2). Each numbered point of such a tetrahedron can serve as the origin of a primitive cubic Bravais lattice. The FCC lattice is then decomposed into four numbered sublattices.

Following [5], in our work we will consider, as a specific illustrative prototype, the alloy $\mathrm{Cu}_{3} \mathrm{Au}$, so that each lattice site is occupied by either a $C u$ or an $A u$ atom. Continuing this emphasis on illustrative special prototypes, we focus on boundary regions between two phases, one of them termed "ordered" (the $\mathrm{Cu}$ and $\mathrm{Au}$ atoms are as illustrated in Fig. 2) and the other totally disordered 


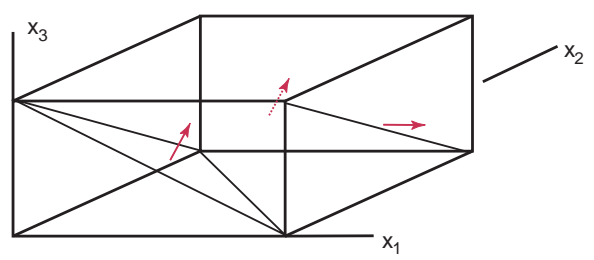

$\operatorname{Lg}_{\bar{n}}=(1,0,0)$
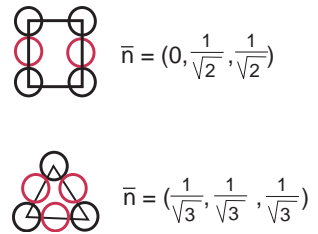

FIG. 1. The distinguished planar cuts.
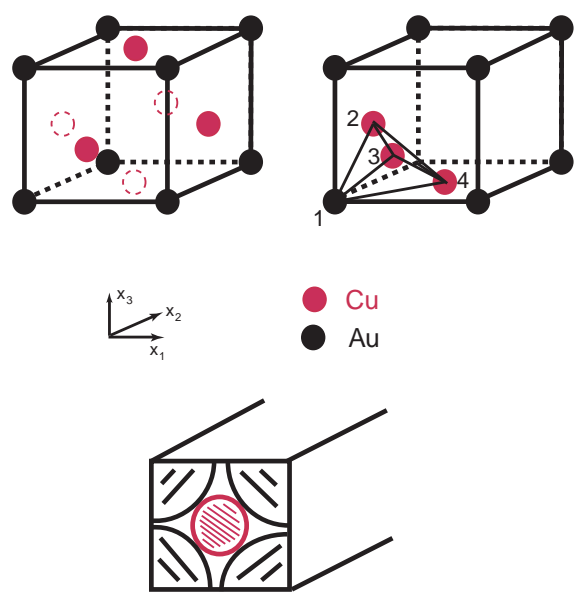

FIG. 2. A unit cell of the FCC lattice, and the tetrahedron whose corners serve to number the four primitive cubic sublattices. The lower schematic indicates a way to visualize the number of $C u$ and $A u$ atoms assigned to each unit cell.

(the assignment of any given site in the unit cell to $C u$ or $A u$ is random, subject to the ratio of $C u$ to $A u$ being the same as in the ordered state).

There will be other occasions as well, during our treatment, when restrictions to special cases are made. The reasons will be mainly to simplify the rigorous analysis. In fact a full mathematical treatment of interphase boundaries in this multiple order parameter phase field scenario appears presently to be an unreasonable goal, at least without a wealth of obfuscating details.

We will focus, at least at first, on interphase boundaries representing order-disorder transitions.

The order parameters. In the ordered form the copper atoms occupy the centers of the faces and the gold atoms the vertices. Four numbers $\rho_{1}, \rho_{2}, \rho_{3}, \rho_{4}$ are defined, when ordering is imperfect, to be the fraction of atoms on each primitive cubic sublattice which are $C u$. When ordering is perfect, copper represents $3 / 4$ of the total. Hence for the ordered $C u_{3} A u$ state,

$$
\rho_{1}(\text { ord })=0, \quad \rho_{2}(\text { ord })=\rho_{3}(\text { ord })=\rho_{4}(\text { ord })=1,
$$

while for the disordered state

$$
\rho_{1}(\text { dis })=\rho_{2}(\text { dis })=\rho_{3}(\text { dis })=\rho_{4}(\text { dis })=3 / 4 \text {. }
$$


In our treatment, the order parameters $\rho_{i}$ are taken to vary continuously within the order-disorder transition region. The equations we will be dealing with are written in terms of the alternative variables $X, Y, Z, W$, defined as linear combinations of the $\rho$ 's:

$$
\begin{aligned}
X & =\frac{1}{4}\left(\rho_{1}+\rho_{2}-\rho_{3}-\rho_{4}\right), \\
Y & =\frac{1}{4}\left(\rho_{1}-\rho_{2}+\rho_{3}-\rho_{4}\right), \\
Z & =\frac{1}{4}\left(\rho_{1}-\rho_{2}-\rho_{3}+\rho_{4}\right), \\
W & =\frac{1}{4}\left(\rho_{1}+\rho_{2}+\rho_{3}+\rho_{4}\right) .
\end{aligned}
$$

The intuition behind this transformation is that the new order parameters are more amenable to continuizing [18]. The first three are nonconserved order parameters and the fourth, $W$, is conserved, since it represents the total density of copper in the crystal. It will be taken fixed (in a more complete model, $W$ would be taken as fixed not pointwise, but only on average [22]). In what follows we choose to redefine the variables $(X, Y, Z, W)$ as multiples of the previous ones by a fixed number. To avoid new notation, we continue to use the previous symbols $(X, Y, Z, W)$ for the new variables. The disordered state now corresponds to

$$
X=Y=Z=0,
$$

and the ordered state to

$$
X=Y=Z=1 .
$$

The free energy. Since $W$ is held fixed, the free energy function used in [5] depends only on $X, Y, Z$ and their gradients:

$$
J(X, Y, Z)=\int_{\Omega}[Q(\nabla X, \nabla Y, \nabla Z)+F(X, Y, Z)] \mathrm{d} \xi_{1} \mathrm{~d} \xi_{2} \mathrm{~d} \xi_{3},
$$

where the space coordinates are $\left(\xi_{1}, \xi_{2}, \xi_{3}\right)$ and $\Omega$ is the volume occupied by the sample. Here $Q$ is a positive definite quadratic form and $F$ is a fourth degree polynomial which is positive except at its several global minima, including $(0,0,0)$ and $(1,1,1)$. The bulk free energy $F$ must conform to certain crystalline symmetries, for instance it must be invariant under permutation of $X, Y, Z$; if it is restricted to be a fourth degree polynomial, its general form is that given below in (3).

The function $Q$ represents the influence on the free energy of the differences between the order parameters at a point and those at nearest neighbors. It is generally the case that this contribution depends on the orientation of the line between these nearby points, and this dependence is a source of anisotropy. The simplest type of quadratic form $Q$ which accounts for anisotropy and which satisfies other symmetry conditions is of the form $Q=A Q_{1}+B Q_{2}$, where $Q_{i}$ are the simple sums of squares of derivatives given below in 4 . The ratio $B / A \equiv \epsilon^{2}$ will then be taken as a measure of the degree of anisotropy of the free energy. In fact, it will be shown that isotropy corresponds to the case $B=A(\epsilon=1)$; our focus will be on the anisotropic case $\epsilon \ll 1$.

As a result, we obtain the following for $F$ and $Q$ :

$$
\begin{aligned}
F(X, Y, Z)= & a_{2}\left(X^{2}+Y^{2}+Z^{2}\right)+a_{3} X Y Z+a_{41}\left(X^{4}+Y^{4}+Z^{4}\right) \\
& +a_{42}\left(X^{2} Y^{2}+X^{2} Z^{2}+Y^{2} Z^{2}\right), \\
Q_{1}= & \frac{1}{2}\left[\left(\frac{\partial X}{\partial \xi_{1}}\right)^{2}+\left(\frac{\partial Y}{\partial \xi_{2}}\right)^{2}+\left(\frac{\partial Z}{\partial \xi_{3}}\right)^{2}\right], \\
Q_{2}= & \frac{1}{2}\left[\left(\frac{\partial X}{\partial \xi_{2}}\right)^{2}+\left(\frac{\partial X}{\partial \xi_{3}}\right)^{2}+\left(\frac{\partial Y}{\partial \xi_{1}}\right)^{2}+\left(\frac{\partial Y}{\partial \xi_{3}}\right)^{2}+\left(\frac{\partial Z}{\partial \xi_{1}}\right)^{2}+\left(\frac{\partial Z}{\partial \xi_{2}}\right)^{2}\right] .
\end{aligned}
$$


The approacandere will be to assume dynamics governed by a gradient flow with respect to $J$, and examine the nature of the interface between grains of ordered and disordered material. In general, these two states will enjoy different bulk free energies, and the interface will migrate. However, the motion depends on the values of the coefficients in 33, which in turn depend on the temperature. The simplest situation is when the two bulk values of $F$ are the same. We assume that the temperature is chosen so that this is the case, and that the two equilibria are $(0,0,0)$ and $(1,1,1)$, representing the disordered and ordered state respectively. A possible choice of the coefficients in (3) such that $F(0,0,0)=F(1,1,1)=0$ is $a_{2}=2, a_{3}=-12, a_{41}=a_{42}=1$. The truncation to fourth degree is discussed in [5] and the extension to sixth degree in [22]. The inclusion of cubic terms is sufficient for the existence of first-order transitions [15].

The phase field equations. The governing evolution PDE's are given by the $L^{2}$ gradient flow of this functional:

$$
\tau \frac{\partial}{\partial t}\left(\begin{array}{l}
X \\
Y \\
Z
\end{array}\right)=L\left(\begin{array}{l}
X \\
Y \\
Z
\end{array}\right)-\nabla F(X, Y, Z),
$$

where $L$ is a diagonal matrix of second degree elliptic operators in the space variables, $\nabla$ denotes the gradient with respect to the variables $(X, Y, Z)$, and $\tau$ is a dimensionless relaxation time.

Although we have described the model in terms of the ordering of a special binary alloy, it serves partially as a pattern for the treatment of other multiphase materials.

The fundamental paper [5], in addition to the derivation of the model, contains a bifurcation analysis of the uniform steady states, numerical and formal asymptotic analyses of plane wave solutions for large anisotropy ratios $A / B \equiv \epsilon^{-2}$, and numerical calculation of the Wulff shapes. This paper together with [22] are our basic references. Other related work is cited in these references. We remark that the parameter $\epsilon$ should not be confused with the usual epsilon appearing before the gradient in the Allen-Cahn and Cahn-Hilliard equations, where it has an entirely different meaning.

Grain boundaries as planar solutions. Plane waves in direction $\bar{n}$ and with velocity $V$ are solutions of (5) of the form

$$
X=x\left(\bar{n} \cdot\left(\xi_{1}, \xi_{2}, \xi_{3}\right)-V t\right)=x(s), \quad Y=y(s), \quad Z=z(s),
$$

with boundary conditions $x(-\infty)=y(-\infty)=z(-\infty)=0, x(\infty)=y(\infty)=z(\infty)=1$. They represent planar interfaces with normal $\bar{n}$ separating an ordered state from a disordered state. The functions $\bar{x}=(x, y, z)$ satisfy (derivatives are with respect to $s)$

$$
-V \bar{x}^{\prime}=\Lambda \bar{x}^{\prime \prime}-\nabla F(x, y, z),
$$

where $\Lambda$ is a diagonal matrix whose elements are linear functions of $A$ and $B$ and quadratic functions of $\bar{n}$.

However, recall that the temperature has been chosen so that the coefficients of $F$ are such that $F$ has equal depth wells at the equilibria of the order-disorder transition: $F(0,0,0)=F(1,1,1)$. We see by taking the scalar product of (7) with $\bar{x}^{\prime}$ and integrating that $V=0$.

\subsection{Symmetries}

Simplifications to the system (7) can be made by seeking only those profiles which satisfy certain symmetry constraints. Doing so reduces the dimensionality of the model to two, thereby making the 
mathematics more tractable, while retaining features characteristic of higher-dimensional models. Of course the direction $\bar{n}$ must be chosen so that the resulting profile satisfies those constraints, and the function $F$ and the matrix $\Lambda$ have to be compatible with them as well.

One such possible constraint is the restriction of the order parameters to the plane $Y=Z$ (hence $y=z$ ). In the crystal, this is tied to the symmetry between two sites on the elementary tetrahedron in Fig. 11 Note from (1) that $Y=Z \Rightarrow \rho_{3}=\rho_{4}$ and that the two symmetric sites share the same $\xi_{1}$ coordinate. Therefore if we take $\bar{n}$ so that $n_{2}=n_{3}$, the symmetry $Y=Z$ should be preserved through the transition. This is indeed true. To exhibit the resulting system, we define our anisotropy parameter $\epsilon=\sqrt{B / A}$; the reduced free energy is

$$
g(x, r)=F\left(x, \frac{1}{\sqrt{2}} r, \frac{1}{\sqrt{2}} r\right)
$$

( $r=\sqrt{2} x=\sqrt{2} y$ is a modified order parameter); and the angle $\alpha$ is defined by $\bar{n}=$ $\left(\cos \alpha, \frac{1}{\sqrt{2}} \sin \alpha, \frac{1}{\sqrt{2}} \sin \alpha\right), 0 \leqslant \alpha \leqslant \pi$. Then 77 is reduced to

$$
\begin{gathered}
\left(\cos ^{2} \alpha+\epsilon^{2} \sin ^{2} \alpha\right) x^{\prime \prime}-g_{x}(x, r)=0, \quad x(-\infty)=r(-\infty)=0, \\
\left(\epsilon^{2} \cos ^{2} \alpha+\frac{1+\epsilon^{2}}{2} \sin ^{2} \alpha\right) r^{\prime \prime}-g_{r}(x, r)=0, \quad x(\infty)=1, \quad r(\infty)=\sqrt{2} .
\end{gathered}
$$

Isotropy means that the coefficients here are independent of the orientation $\bar{n}$, i.e. of $\alpha$. This happens when $\epsilon=1$; our main interest is in the extreme anisotropic case $\epsilon \ll 1$.

As can be easily verified, the gradient flow (5) that determines the full dynamics of the phase field model leaves invariant the subspace of functions

$$
S=\left\{\left(X\left(\xi_{1}, \xi_{2}, \xi_{3}\right), Y\left(\xi_{1}, \xi_{2}, \xi_{3}\right), Z\left(\xi_{1}, \xi_{2}, \xi_{3}\right)\right): Y=Z\right\}
$$

Note that the special cases $\alpha=0$ and $\alpha=\pi / 2$ correspond to the distinguished cuts $(1,0,0)$ and $(0,1 / \sqrt{2}, 1 / \sqrt{2})$ already mentioned above. When $\epsilon \ll 1$, the problem for the profile at $\alpha=0$ or $\alpha=\pi / 2$ is a singular perturbation problem from a limit profile at $\epsilon=0$, "singular" because passing to the limit $\epsilon=0$ reduces the order of the system 10 from 4 to 2 . In the case $\alpha=0$, the problem is particularly difficult because of a degeneracy (irregularity) in the limit profile which, for example, does not allow for the application of the Fenichel theory and its variants.

\subsection{Statement of results}

In the present paper we study the orientation $\alpha=\pi / 2$ corresponding to grain boundaries parallel to the second distinguished cut in Fig. 1. We establish existence and spectral stability of the interface between the ordered and disordered states in the invariant subspace $S$, i.e. we do this for solutions of (9). The case $\alpha=0$ is very different both in results and methods of proof. We refer to [1], [9].

More precisely, we prove the following theorems (see the remarks following (11)):

THEOREM A (The heteroclinic orbit) If $\epsilon>0$ is sufficiently small, then there exists a solution $\left(x_{\epsilon}(s), r_{\epsilon}(s)\right)$ of (11) such that:

$$
\begin{gathered}
x_{\epsilon}=x_{0}+\tilde{x}_{\epsilon}, \quad r_{\epsilon}=r_{0}+\tilde{r}_{\epsilon} \quad \text { with } \tilde{x}_{\epsilon}, \tilde{r}_{\epsilon} \in H^{2}(\mathbb{R}) \cap C^{2}(\mathbb{R}), \\
\left(\tilde{x}_{\epsilon}, \tilde{r}_{\epsilon}\right) \perp\left(x_{0}^{\prime}, r_{0}^{\prime}\right) \quad\left(\text { in } L^{2}(\mathbb{R}) \times L^{2}(\mathbb{R})\right),
\end{gathered}
$$

and

$$
\left\|\left(\tilde{x}_{\epsilon}, \tilde{r}_{\epsilon}\right)\right\|_{1, \epsilon}=O\left(\epsilon^{2}\right)
$$


where

$$
\left\|\left(\tilde{x}_{\epsilon}, \tilde{r}_{\epsilon}\right)\right\|_{1, \epsilon}^{2}=\epsilon^{2}\left\|\tilde{x}_{\epsilon}^{\prime}\right\|_{L^{2}}^{2}+\left\|\tilde{r}_{\epsilon}^{\prime}\right\|_{L^{2}}^{2}+\left\|\tilde{x}_{\epsilon}\right\|_{L^{2}}^{2}+\left\|\tilde{r}_{\epsilon}\right\|_{L^{2}}^{2} .
$$

Here $\left(x_{0}(s), r_{0}(s)\right)$ is the solution of 9 for $\epsilon=0$, defined below in Sec.2.2

THEOREM B (Stability of spectrum) Let $\left(x_{\epsilon}, r_{\epsilon}\right)$ be the heteroclinic orbit of Theorem A. Then if $\epsilon>0$ is sufficiently small, the spectrum of the linearized operator $L_{\epsilon}$ about $\left(x_{\epsilon}, r_{\epsilon}\right)$ has the following form: zero is a simple eigenvalue at the bottom of the spectrum and the rest of the spectrum is contained in $[C, \infty)$, where $C$ is a positive constant independent of $\epsilon$.

As we have mentioned before, existence for (9), $\alpha=\pi / 2$, can be treated via Fenichel's invariant manifold theory [8]. For this choice of $\alpha, \sqrt{9}]$ is equivalent, by simple stretching of variables, to

$$
\begin{aligned}
\epsilon^{2} x^{\prime \prime} & =g_{x}(x, r), \\
r^{\prime \prime} & =g_{r}(x, r) .
\end{aligned}
$$

This system is Hamiltonian and for $\epsilon=0$ it has a heteroclinic connection $\left(x_{0}(s), r_{0}(s)\right)$, a standard fact.

The limit case $\epsilon=0$ has an easily obtained solution. Theorems A and B are in effect singular perturbation results corresponding to small positive $\epsilon$. The first asserts that when there is a large amount of anisotropy, a resulting phase interface exists and is a small perturbation of the ideal limiting one. Moreover, the change in the interfacial profiles $x, x^{\prime}, r, r^{\prime}$ can be estimated in terms of that singular perturbation parameter $\epsilon$.

Theorem B is a robustness result for the profile, which begins to address the effects of a multitude of physical influences which may affect the phase field model. Among these are departures from symmetry, the motion of interfaces due to temperature changes and to their possible curvature, and many other deficiencies in the modeling. What Theorem B says, in a very general way, is that due to the stability of the spectrum, such influences which may disrupt the special conditions under which the profile's existence was proved are not, if they are small, expected to change either the existence result or the properties of the interface, in other than a minor way. Since every mathematical model in this field of study is necessarily only an approximately valid conception, questions of robustness form a central issue. The stability of the spectrum is a natural first place to begin an investigation of robustness. Further steps would involve modeling the disruptions themselves; but the set of possible disruptions is vast and largely unknown, so those steps would be a formidable undertaking inappropriate for a paper like this.

Fenichel's machinery, as presented for example in Jones [14], allows the reduction of the existence problem to one on a two-dimensional center-like manifold, and by using the Hamiltonian structure one can establish the existence of a heteroclinic connection for small $\epsilon>0$.

The main approach to the question of existence in this paper is different from Fenichel's, and more appropriate to the robustness issues mentioned above. Our procedure is based on the study of the linearized operator of the parabolic problem (5p), constrained by the symmetries already mentioned, about the zero order approximation to the heteroclinic. Thus it deals with an infinitedimensional phase space and is more functional-analytic. The main effort is to establish a theorem on the spectrum of this operator. Then this result is utilized in Sec. 3, where the existence of the heteroclinic is established fairly quickly via a contraction mapping argument. Finally, in Sec. 4 we establish stability of the spectrum of the heteroclinic via the theorem in Sec. 2 and a simple perturbation argument. 


\section{The spectrum}

\subsection{Preliminaries}

The boundary value problem (11) to be considered is

$$
\begin{aligned}
& \epsilon^{2} x^{\prime \prime}=g_{x}(x, r), \\
& r^{\prime \prime}=g_{r}(x, r), \\
& x=x(s), \quad r=r(s), \quad s \in \mathbb{R}, \quad '=\mathrm{d} / \mathrm{d} s, \\
& x(-\infty)=r(-\infty)=0, \quad x(\infty)=1, \quad r(\infty)=\sqrt{2} .
\end{aligned}
$$

For future reference we record here the most relevant facts about the function $g$. From (8), (3),

$$
g(x, r)=2\left(x^{2}+r^{2}\right)-6 x r^{2}+x^{2} r^{2}+\frac{3}{4} r^{4}+x^{4},
$$

with equal minima at $(0,0)$ and $(1, \sqrt{2})$. Therefore

$$
\begin{gathered}
g_{x}=4 x-6 r^{2}+2 x r^{2}+4 x^{3}, \quad g_{r}=r\left(4-12 x+2 x^{2}+3 r^{2}\right), \\
g_{x x}=4+2 r^{2}+12 x^{2} \geqslant 4, \\
g_{x r}=-12 r+4 x r \\
g_{r r}=4-12 x+2 x^{2}+9 r^{2} .
\end{gathered}
$$

Furthermore,

$$
g_{x}=0 \Leftrightarrow r^{2}=\frac{2 x+2 x^{3}}{3-x},
$$

which can be inverted for $x \in[0,3)$ to obtain $x=\chi(r)$, where $\chi: \mathbb{R} \rightarrow[0,3)$ is smooth, even, and $\chi(0)=0, \chi(\sqrt{2})=1, \chi^{\prime}(0)=0$.

\subsection{The $\epsilon=0$ approximation}

Setting $\epsilon=0$ in the first equation of (12) and using the expectation that $x(s) \in[0,1]$, we get $x=\chi(r)$. Substituting into the second equation, we have

$$
r^{\prime \prime}-g_{r}(\chi(r), r)=0
$$

We look for a solution to this equation such that $r(-\infty)=0, r(+\infty)=\sqrt{2}$.

Setting

$$
G(r)=g(\chi(r), r), \quad r \in \mathbb{R}
$$

we have

$$
G^{\prime}(r)=g_{x}(\chi(r), r) \chi^{\prime}(r)+g_{r}(\chi(r), r)=g_{r}(\chi(r), r) .
$$

Thus (19) can be written as

$$
r^{\prime \prime}-G^{\prime}(r)=0
$$


It can be checked from $\sqrt{16}),(18)$ that as $r$ ranges from 0 to $\sqrt{2}$, the function $G^{\prime}(r)=g_{r}(\chi(r), r)$ starts positive and then becomes negative. Also $G(0)=G(\sqrt{2})=0$.

It is known [4, 11] that in the phase plane there is a heteroclinic orbit connecting $(0,0)$ to $(\sqrt{2}, 0)$. This furnishes a solution to 19 with the desired behavior at infinity, unique up to translation. We denote it by $r_{0}(s)$ and call the "base heteroclinic".

We also know that $r_{0}^{\prime}(s)>0$ for $s \in \mathbb{R}$, hence $0<r_{0}(s)<\sqrt{2}$. We set $x_{0}(s)=\chi\left(r_{0}(s)\right)$. Then $x_{0}(-\infty)=\chi\left(r_{0}(-\infty)\right)=\chi(0)=0, x_{0}(\infty)=\chi(\sqrt{2})=1$. Hence the pair $\left(x_{0}, r_{0}\right)$ is a solution of 121-14] for $\epsilon=0$. Furthermore, we know that $r_{0}(s)$ and $r_{0}^{\prime}(s)$ approach their limits exponentially as $s \rightarrow \pm \infty$. This follows from $G^{\prime \prime}(0)>0, G^{\prime \prime}(\sqrt{2})>0$ via linear theory.

From $\sqrt{22}$ we then deduce that $r_{0}^{\prime \prime}$ converges exponentially as $|s| \rightarrow \infty$ to 0 . Also $r_{0}^{\prime \prime \prime}=$ $r_{0}^{\prime} G^{\prime \prime}\left(r_{0}\right)$, which implies that $r_{0}^{\prime \prime \prime}$ converges exponentially to 0 .

Function spaces and the bistable operator. In this paper the notation $L^{2}, H^{2}, C^{2}$ will denote the corresponding spaces of functions defined on the whole real line $\mathbb{R}: L^{2}(\mathbb{R})$, etc.

From the exponential convergence, we know that $r_{0}^{\prime}, r_{0}^{\prime \prime}, r_{0}^{\prime \prime \prime} \in L^{2}$, which in turn gives that $r_{0}^{\prime} \in H^{2}$. Similarly, since $x_{0}=\chi\left(r_{0}\right)$, we get $x_{0}^{\prime} \in H^{2}$.

We also note that for $r \in \mathbb{R}$,

$$
G^{\prime \prime}(r)=g_{x r}(\chi(r), r) \chi^{\prime}(r)+g_{r r}(\chi(r), r)=-\frac{\left[g_{x r}(\chi(r), r)\right]^{2}}{g_{x x}(\chi(r), r)}+g_{r r}(\chi(r), r) .
$$

Set

$$
q(s) \doteq G^{\prime \prime}\left(r_{0}(s)\right), \quad s \in \mathbb{R} .
$$

We define the operator $B$ by

$$
B h=-h^{\prime \prime}+q h \quad \text { with } D(B)=H^{2},
$$

which is unbounded and self-adjoint on $L^{2}$. It is called the bistable operator. The following facts are well known:

- The essential spectrum of $B$ is contained in $\left[C_{1}, \infty\right)$, where $C_{1}>0$.

- The smallest eigenvalue of $B$ is 0 , which is simple (we know that $r_{0}^{\prime} \in \operatorname{ker}(B)$ ).

Hence by the variational characterization of the eigenvalues of self-adjoint operators (see [20]) we have

$$
\int_{-\infty}^{\infty}\left[\left(h^{\prime}\right)^{2}+q h^{2}\right] \mathrm{d} s \geqslant C_{2}\|h\|_{L^{2}}^{2} \quad \text { for all } h \in H^{2}, h \perp r_{0}^{\prime}\left(\text { in } L^{2}\right) .
$$

\subsection{The operator $L_{\epsilon}$}

We now consider the operator $L_{\epsilon}$, obtained by linearizing (12) about $\left(x_{0}, r_{0}\right)$. We have

$$
L_{\epsilon}\left(\begin{array}{l}
h_{1} \\
h_{2}
\end{array}\right)=\left(\begin{array}{c}
-\epsilon^{2} h_{1}^{\prime \prime}+g_{x x}\left(x_{0}, r_{0}\right) h_{1}+g_{x r}\left(x_{0}, r_{0}\right) h_{2} \\
-h_{2}^{\prime \prime}+g_{x r}\left(x_{0}, r_{0}\right) h_{1}+g_{r r}\left(x_{0}, r_{0}\right) h_{2}
\end{array}\right),
$$

with $L_{\epsilon}: D\left(L_{\epsilon}\right) \rightarrow L^{2} \times L^{2}, D\left(L_{\epsilon}\right)=H^{2} \times H^{2}$. It is an unbounded self-adjoint operator in $L^{2} \times L^{2}$. 
We recall that $\left(x_{0}(s), r_{0}(s)\right) \rightarrow(0,0)$ as $s \rightarrow-\infty$ and $\left(x_{0}(s), r_{0}(s)\right) \rightarrow(1, \sqrt{2})$ as $s \rightarrow+\infty$ (in all cases, exponentially); and that (see (17))

$$
\begin{aligned}
& g_{x x}(0,0)=4, \quad g_{x r}(0,0)=0, \quad g_{r r}(0,0)=4, \\
& g_{x x}(1, \sqrt{2})=20, \quad g_{x r}(1, \sqrt{2})=-8 \sqrt{2}, \quad g_{r r}(1, \sqrt{2})=12 .
\end{aligned}
$$

We write

$$
D_{\epsilon}=\left(\begin{array}{cc}
\epsilon^{2} & 0 \\
0 & 1
\end{array}\right), \quad N(s)=\left(\begin{array}{ll}
g_{x x}\left(x_{0}, r_{0}\right) & g_{x r}\left(x_{0}, r_{0}\right) \\
g_{x r}\left(x_{0}, r_{0}\right) & g_{r r}\left(x_{0}, r_{0}\right)
\end{array}\right)
$$

so that

$$
N(-\infty)=\left(\begin{array}{ll}
4 & 0 \\
0 & 4
\end{array}\right), \quad N(+\infty)=\left(\begin{array}{cc}
20 & -8 \sqrt{2} \\
-8 \sqrt{2} & 12
\end{array}\right)
$$

Then

$$
L_{\epsilon} h=-D_{\epsilon} h^{\prime \prime}+N(s) h, \quad h=\left(h_{1}, h_{2}\right)^{\perp} .
$$

In what follows, we make use of the operator

$$
\tilde{L}_{\epsilon} h=-D_{\epsilon} h^{\prime \prime}+\tilde{N}(s) h,
$$

where $\tilde{N}(s)=N(-\infty)$ if $s \leqslant 0$, and $\tilde{N}(s)=N(\infty)$ if $s>0$. We can easily show that

$$
\left(\tilde{L}_{\epsilon} h, h\right)_{L^{2} \times L^{2}} \geqslant C_{3}\|h\|_{L^{2} \times L^{2}}^{2} \quad \forall h \in H^{2} \times H^{2},
$$

with $C_{3}>0$ independent of $\epsilon>0$. Hence

$$
\sigma_{\mathrm{ess}}\left(\tilde{L}_{\epsilon}\right) \subset \sigma\left(\tilde{L}_{\epsilon}\right) \subset\left[C_{3}, \infty\right)
$$

Also $\left(L_{\epsilon}-\tilde{L}_{\epsilon}\right) h=(N(s)-\tilde{N}(s)) h$, where $N(s)-\tilde{N}(s) \rightarrow 0$ in the matrix norm exponentially as $|s| \rightarrow \infty$.

It can be shown that the operator

$$
\left(L_{\epsilon}-\tilde{L}_{\epsilon}\right) \tilde{L}_{\epsilon}^{-1}: L^{2} \times L^{2} \rightarrow L^{2} \times L^{2}
$$

is compact. In other words, $L_{\epsilon}-\tilde{L}_{\epsilon}$ is relatively $\tilde{L}_{\epsilon}$-compact. From this it follows that

$$
\sigma_{\mathrm{ess}}\left(L_{\epsilon}\right)=\sigma_{\mathrm{ess}}\left(\tilde{L}_{\epsilon}\right) \subset\left[C_{3}, \infty\right)
$$

([13, p. 140]).

Occasionally we will drop the subscript $\epsilon$ and write $L$ instead of $L_{\epsilon}$.

Throughout this paper, all the inner products are in the sense of $L^{2}$ or $L^{2} \times L^{2}$, unless specified otherwise. The $L^{2} \times L^{2}$ inner product is defined by $(u, v)_{L^{2} \times L^{2}}=\int_{-\infty}^{\infty}\left(u_{1} v_{1}+u_{2} v_{2}\right) \mathrm{d} s$, where $u=\left(u_{1}, u_{2}\right), v=\left(v_{1}, v_{2}\right) \in L^{2} \times L^{2}$. Furthermore, all the constants $C_{i}$ will be independent of $\epsilon>0$. 
2.4 The operators $K_{1}^{\epsilon}(\lambda), K_{2}^{\epsilon}(\lambda)$, and the reduction to a single equation with bistable structure

The eigenvalues $\lambda$ of $L$ and their corresponding eigenfunctions $\left(h_{1}, h_{2}\right) \in H^{2} \times H^{2}$ satisfy

$$
\begin{gathered}
-\epsilon^{2} h_{1}^{\prime \prime}(s)+g_{x x}\left(x_{0}(s), r_{0}(s)\right) h_{1}(s)+g_{x r}\left(x_{0}(s), r_{0}(s)\right) h_{2}(s)=\lambda h_{1}(s) \\
-h_{2}^{\prime \prime}(s)+g_{x r}\left(x_{0}(s), r_{0}(s)\right) h_{1}(s)+g_{r r}\left(x_{0}(s), r_{0}(s)\right) h_{2}(s)=\lambda h_{2}(s) \\
\left\|h_{1}\right\|_{L^{2}}^{2}+\left\|h_{2}\right\|_{L^{2}}^{2}=1
\end{gathered}
$$

From now on for simplicity we will not explicitly write $s$ and write instead $g_{x x}$ in place of $g_{x x}\left(x_{0}(s), r_{0}(s)\right)$, etc. Also since we are interested in the small eigenvalues of $L$, we can assume without loss of generality that $\lambda \leqslant 2$.

From (36) we have

$$
-\epsilon^{2} h_{1}^{\prime \prime}+\left(g_{x x}-\lambda\right) h_{1}=-g_{x r} h_{2} .
$$

We know that $g_{x x} \geqslant 4$ and since $\lambda \leqslant 2$, we have $g_{x x}-\lambda \geqslant 2$. Hence for each $\epsilon>0, \lambda \leqslant 2$, and $h_{2} \in L^{2}$, there exists a unique solution $h_{1} \in H^{2}$ of the above equation. Hence we can define a linear operator $K_{1}^{\epsilon}(\lambda): L^{2} \rightarrow L^{2}$ by

$$
h_{1} \doteq K_{1}^{\epsilon}(\lambda) h_{2}
$$

for $\epsilon>0, \lambda \leqslant 2$. We also define another linear operator $K_{2}^{\epsilon}(\lambda): H^{2} \rightarrow H^{2}$ for $\epsilon>0$ and $\lambda \leqslant 2$ by writing

$$
h_{1}=K_{1}^{\epsilon}(\lambda) h_{2}=-\frac{g_{x r}}{g_{x x}-\lambda} h_{2}+\epsilon^{2} K_{2}^{\epsilon}(\lambda) h_{2},
$$

where

$$
K_{2}^{\epsilon}(\lambda) h_{2} \doteq \frac{1}{\epsilon^{2}}\left(\frac{g_{x r}}{g_{x x}-\lambda} h_{2}+K_{1}^{\epsilon}(\lambda) h_{2}\right)
$$

Observe that the first term on the right side of (41) is the solution $h_{1}$ of (39) when $\epsilon=0$. Substituting (41) into 37), we get

$$
-h_{2}^{\prime \prime}+g_{x r}\left[-\frac{g_{x r}}{g_{x x}-\lambda} h_{2}+\epsilon^{2} K_{2}^{\epsilon}(\lambda) h_{2}\right]+g_{r r} h_{2}=\lambda h_{2},
$$

which we rewrite in the form

$$
-h_{2}^{\prime \prime}+\left(g_{r r}-\frac{g_{x r}^{2}}{g_{x x}}\right) h_{2}=\lambda\left[1+\frac{g_{x r}^{2}}{g_{x x}\left(g_{x x}-\lambda\right)}\right] h_{2}-\epsilon^{2} g_{x r} K_{2}^{\epsilon}(\lambda) h_{2} .
$$

Hence the eigenvalue problem (36) -38 is equivalent to

$$
\begin{gathered}
h_{1}=K_{1}^{\epsilon}(\lambda) h_{2}, \\
-h_{2}^{\prime \prime}+\left(g_{r r}-\frac{g_{x r}^{2}}{g_{x x}}\right) h_{2}=\lambda\left[1+\frac{g_{x r}^{2}}{g_{x x}\left(g_{x x}-\lambda\right)}\right] h_{2}-\epsilon^{2} g_{x r} K_{2}^{\epsilon}(\lambda) h_{2}, \\
\left\|h_{1}\right\|_{L^{2}}^{2}+\left\|h_{2}\right\|_{L^{2}}^{2}=1 .
\end{gathered}
$$

Observe that on the left of (45), we have the bistable operator $B$ of (25). 


\section{$2.5 \quad H^{2}$ estimates}

LEMma 1 For every $\lambda, \lambda_{1}, \lambda_{2} \leqslant 2$ and every $\epsilon>0$, the following inequalities hold:

$$
\begin{aligned}
\left\|K_{1}^{\epsilon}(\lambda) f\right\|_{L^{2}} & \leqslant C_{4}\|f\|_{L^{2}} \quad \forall f \in L^{2}, \\
\left\|K_{2}^{\epsilon}(\lambda) f\right\|_{L^{2}} & \leqslant C_{5}\|f\|_{H^{2}} \quad \forall f \in H^{2}, \\
\left\|\left(K_{2}^{\epsilon}\left(\lambda_{1}\right)-K_{2}^{\epsilon}\left(\lambda_{2}\right)\right) f\right\|_{L^{2}} & \leqslant C_{6}\left|\lambda_{1}-\lambda_{2}\right|\|f\|_{H^{2}} \quad \forall f \in H^{2},
\end{aligned}
$$

where $C_{4}, C_{5}, C_{6}$ are independent of $\lambda, \lambda_{1}, \lambda_{2}, \epsilon$ and $f$.

Proof. Let $f \in L^{2}, \lambda \leqslant 2$ and $\epsilon>0$, and set $X=K_{1}^{\epsilon}(\lambda) f \in H^{2}$. Then by the definition of the linear operator $K_{1}^{\epsilon}(\lambda)$, we have

$$
-\epsilon^{2} X^{\prime \prime}+\left(g_{x x}-\lambda\right) X=-g_{x r} f
$$

from which we obtain

$$
-\epsilon^{2} \int_{-\infty}^{\infty} X^{\prime \prime} X \mathrm{~d} s+\int_{-\infty}^{\infty}\left(g_{x x}-\lambda\right) X^{2} \mathrm{~d} s=-\int_{-\infty}^{\infty} g_{x r} f X \mathrm{~d} s,
$$

and by integration by parts, which is valid since $X \in H^{2}$, we get

$$
\epsilon^{2} \int_{-\infty}^{\infty}\left(X^{\prime}\right)^{2} \mathrm{~d} s+\int_{-\infty}^{\infty}\left(g_{x x}-\lambda\right) X^{2} \mathrm{~d} s=-\int_{-\infty}^{\infty} g_{x r} f X \mathrm{~d} s .
$$

(we also used the fact that $X(s), X^{\prime}(s) \rightarrow 0$ as $|s| \rightarrow \infty$, since $X \in H^{2}$ ). Now since $g_{x x}-\lambda \geqslant 2$ and $g_{x r}$ is bounded, by the Cauchy-Schwarz inequality we have

$$
2\|X\|_{L^{2}}^{2} \leqslant \sup _{s}\left|g_{x r}\right| \int_{-\infty}^{\infty}|f||X| \mathrm{d} s \leqslant \sup _{s \in \mathbb{R}}\left|g_{x r}\right|\|f\|_{L^{2}}\|X\|_{L^{2}} .
$$

Hence $\|X\|_{L^{2}} \leqslant C_{4}\|f\|_{L^{2}}$. This proves (47).

Now let $f \in H^{2}$. By the definition of $K_{2}^{\epsilon}(\lambda)$, the equation

$$
-\epsilon^{2} u^{\prime \prime}+\left(g_{x x}-\lambda\right) u=-g_{x r} f
$$

is equivalent to

$$
u=-\frac{g_{x r}}{g_{x x}-\lambda} f+\epsilon^{2} K_{2}^{\epsilon}(\lambda) f .
$$

Set $X=K_{2}^{\epsilon}(\lambda) f$; then

$$
-\epsilon^{2}\left(-\frac{g_{x r}}{g_{x x}-\lambda} f+\epsilon^{2} X\right)^{\prime \prime}+\left(g_{x x}-\lambda\right)\left(-\frac{g_{x r}}{g_{x x}-\lambda} f+\epsilon^{2} X\right)=-g_{x r} f,
$$

which simplifies to

$$
-\epsilon^{2} X^{\prime \prime}+\left(g_{x x}-\lambda\right) X=-\left(\frac{g_{x r}}{g_{x x}-\lambda} f\right)^{\prime \prime}
$$


By working as before, we get

$$
\|X\|_{L^{2}} \leqslant \frac{1}{2}\left\|\left(\frac{g_{x r}}{g_{x x}-\lambda} f\right)^{\prime \prime}\right\|_{L^{2}}
$$

Taking into account that $g_{x x}-\lambda \geqslant 2$ and that the functions $g_{x x}$ and $g_{x r}$ of $s$ are bounded along with their first and second derivatives, we can estimate the right hand side of the above inequality, and get $\|X\|_{L^{2}} \leqslant C_{5}\|f\|_{H^{2}}$. This proves (48).

Set $X_{1}=K_{2}^{\epsilon}\left(\lambda_{1}\right) f$ and $X_{2}=K_{2}^{\epsilon}\left(\lambda_{2}\right) f$. Then

$$
-\epsilon^{2} X_{i}^{\prime \prime}+\left(g_{x x}-\lambda_{i}\right) X_{i}=-\left(\frac{g_{x r} f}{g_{x x}-\lambda_{i}}\right)^{\prime \prime}, \quad i=1,2,
$$

and so

$$
-\epsilon^{2}\left(X_{1}-X_{2}\right)^{\prime \prime}+\left(g_{x x}-\lambda_{1}\right)\left(X_{1}-X_{2}\right)=\left(\lambda_{1}-\lambda_{2}\right) X_{2}-\left(\frac{g_{x r} f}{g_{x x}-\lambda_{1}}-\frac{g_{x r} f}{g_{x x}-\lambda_{2}}\right)^{\prime \prime} .
$$

Now by (48), we have

$$
\left\|X_{2}\right\|_{L^{2}}=\left\|K_{2}^{\epsilon}\left(\lambda_{2}\right) f\right\|_{L^{2}} \leqslant C_{5}\|f\|_{H^{2}},
$$

and so by working as in the proof of (47) and of (48) (to estimate the norm of the last term of (54)), we get

$$
2\left\|X_{1}-X_{2}\right\|_{L^{2}} \leqslant C_{5}\left|\lambda_{1}-\lambda_{2}\right|\|f\|_{H^{2}}+\left\|\left(\frac{g_{x r} f}{g_{x x}-\lambda_{1}}-\frac{g_{x r} f}{g_{x x}-\lambda_{2}}\right)^{\prime \prime}\right\|_{L^{2}} \leqslant C_{6}\left|\lambda_{1}-\lambda_{2}\right|\|f\|_{H^{2}} .
$$

This proves 499. The proof of Lemma 1 is complete.

Lemma 2 Define, as always, $q=g_{r r}-g_{x r}^{2} / g_{x x}$. If $-h^{\prime \prime}+q h=f, h \in H^{2}, h \perp r_{0}^{\prime}$ (in $L^{2}$ ) and $f \in L^{2}$, then

$$
\|h\|_{H^{2}} \leqslant C_{7}\|f\|_{L^{2}}
$$

with $C_{7}$ independent of $f$.

Proof. Working as in the proof of Lemma1 1 , we get

$$
\int_{-\infty}^{\infty}\left[\left(h^{\prime}\right)^{2}+q h^{2}\right] \mathrm{d} s=\int_{-\infty}^{\infty} f h \mathrm{~d} s \leqslant\|f\|_{L^{2}}\|h\|_{L^{2}} .
$$

Utilizing [26], we have

$$
C_{2}\|h\|_{L^{2}}^{2} \leqslant\|f\|_{L^{2}}\|h\|_{L^{2}}
$$

Hence

$$
\|h\|_{L^{2}} \leqslant C_{2}^{-1}\|f\|_{L^{2}} .
$$

Also,

$$
\left\|h^{\prime \prime}\right\|_{L^{2}}=\|q h-f\|_{L^{2}} \leqslant\|q\|_{\infty}\|h\|_{L^{2}}+\|f\|_{L^{2}} \leqslant\left(\|q\|_{\infty} C_{2}^{-1}+1\right)\|f\|_{L^{2}} .
$$

Obviously $-2 h h^{\prime \prime} \leqslant h^{2}+\left(h^{\prime \prime}\right)^{2}$, and

$$
2 \int_{-\infty}^{\infty}\left(h^{\prime}\right)^{2} \mathrm{~d} s \leqslant\|h\|_{L^{2}}^{2}+\left\|h^{\prime \prime}\right\|_{L^{2}}^{2} .
$$

From (55) and $\left[56\right.$, we obtain $\|h\|_{H^{2}} \leqslant C_{7}\|f\|_{L^{2}}$, which proves the lemma. 


\subsection{A priori estimates}

Proposition 1 Suppose that $(\lambda, h)$ is a solution to $(36)-(38)$. Then there exist positive constants $\epsilon_{0}, \lambda_{0}<\min \left\{2, C_{3}\right\}, M_{0}, a_{0}$, independent of $\epsilon, \lambda, h$, such that if $0<\epsilon<\epsilon_{0}$ and $|\lambda|<\lambda_{0}$, then the following estimates hold:

(i) $|\lambda| \leqslant M_{0} \epsilon^{2}$,

(ii) $\|p\|_{H^{2}} \leqslant M_{0} \epsilon^{2},|a| \geqslant a_{0}$, where

$$
\left.h_{2}=a y+p, \quad y=r_{0}^{\prime} /\left\|r_{0}^{\prime}\right\|_{L^{2}}, \quad p \perp r_{0}^{\prime} \quad \text { (in } L^{2}\right) .
$$

(For simplicity we do not write the dependence of $\lambda, a, p$ on $\epsilon$.)

Proof. Throughout the proof, $C_{i}>0$ will denote constants independent of $\epsilon, \lambda, h$. Substitute $h_{2}=$ $a y+p$ in (45) and set

$$
\tilde{K}_{2}^{\epsilon}(\lambda):=g_{x r} K_{2}^{\epsilon}(\lambda), \quad \sigma:=1+\frac{g_{x r}^{2}}{g_{x x}\left(g_{x x}-\lambda\right)},
$$

with $|\sigma(s)| \leqslant\|\sigma\|_{\infty}, s \in \mathbb{R}\left(\|\sigma\|_{\infty}\right.$ independent of $\lambda$ since $\left.g_{x x}-\lambda \geqslant 2\right)$. Then (45) takes the following form (recall that $-y^{\prime \prime}+q y=0$ ):

$$
-p^{\prime \prime}+q p=\lambda a \sigma y-\epsilon^{2} a \tilde{K}_{2}^{\epsilon}(\lambda) y+\lambda \sigma p-\epsilon^{2} \tilde{K}_{2}^{\epsilon}(\lambda) p .
$$

By Lemma 1, we have

$$
\begin{gathered}
\left\|\tilde{K}_{2}^{\epsilon}(\lambda) f\right\|_{L^{2}} \leqslant C_{8}\|f\|_{H^{2}}, \\
\left\|\tilde{K}_{2}^{\epsilon}\left(\lambda_{1}\right) f-\tilde{K}_{2}^{\epsilon}\left(\lambda_{2}\right) f\right\|_{L^{2}} \leqslant C_{8}\left|\lambda_{1}-\lambda_{2}\right|\|f\|_{H^{2}}
\end{gathered}
$$

for every $f \in H^{2}, \lambda, \lambda_{1}, \lambda_{2} \leqslant 2$ and $\epsilon>0\left(C_{8}\right.$ independent of $\left.\lambda, \lambda_{1}, \lambda_{2}, \epsilon, f\right)$. Multiplying 57 by $y$, integrating, and using the fact that $\left(-p^{\prime \prime}+q p, y\right)_{L^{2}}=\left(p,-y^{\prime \prime}+q y\right)_{L^{2}}=0$ gives

$$
\lambda a(\sigma y, y)-\epsilon^{2} a\left(\tilde{K}_{2}^{\epsilon}(\lambda) y, y\right)+\lambda(\sigma p, y)-\epsilon^{2}\left(\tilde{K}_{2}^{\epsilon}(\lambda) p, y\right)=0 .
$$

Since the function $\sigma$ satisfies $\sigma>1,\|y\|_{L^{2}}=1$, via (58) we have

$$
|\lambda||a| \leqslant \epsilon^{2}|a| C_{8}\|y\|_{H^{2}}+|\lambda|\|\sigma\|_{\infty}\|p\|_{L^{2}}+\epsilon^{2} C_{8}\|p\|_{H^{2}} .
$$

Applying Lemma2 to [57) gives

$$
\begin{aligned}
\|p\|_{H^{2}} & \leqslant C_{7}\left\|\lambda a \sigma y-\epsilon^{2} a \tilde{K}_{2}^{\epsilon}(\lambda) y+\lambda \sigma p-\epsilon^{2} \tilde{K}_{2}^{\epsilon}(\lambda) p\right\|_{L^{2}} \\
& \leqslant C_{7}\left(|\lambda||a|\|\sigma\|_{\infty}+\epsilon^{2}|a| C_{8}\|y\|_{H^{2}}+|\lambda|\|\sigma\|_{\infty}\|p\|_{L^{2}}+\epsilon^{2} C_{8}\|p\|_{H^{2}}\right),
\end{aligned}
$$

or in a more compact form,

$$
\|p\|_{H^{2}} \leqslant C_{9}\left(|\lambda||a|+\epsilon^{2}|a|+|\lambda|\|p\|_{H^{2}}+\epsilon^{2}\|p\|_{H^{2}}\right) .
$$

Choosing $\epsilon_{0}$ and $\lambda_{0}$ such that $C_{9}\left(\lambda_{0}+\epsilon_{0}^{2}\right)<1 / 2$, we get

$$
\|p\|_{H^{2}} \leqslant 2 C_{9}\left(|\lambda||a|+\epsilon^{2}|a|\right) .
$$


Now using Lemma 1. we have $\left\|h_{1}\right\|_{L^{2}}=\left\|K_{1}^{\epsilon}(\lambda) h_{2}\right\|_{L^{2}} \leqslant C_{4}\left\|h_{2}\right\|_{L^{2}}$, so by 46 we have $\left\|h_{2}\right\|_{L^{2}}^{2} \geqslant C_{10}>0$ for $C_{10}=1 /\left(1+C_{4}^{2}\right)$. Also

$$
1 \geqslant\left\|h_{2}\right\|_{L^{2}}^{2}=|a|^{2}+\|p\|_{L^{2}}^{2} \geqslant C_{10}>0 .
$$

In particular, $|a| \leqslant 1$. Thus from (61) we have

$$
\|p\|_{H^{2}} \leqslant 2 C_{9}\left(|\lambda|+\epsilon^{2}\right) .
$$

Further reducing $\epsilon_{0}, \lambda_{0}$ so that $C_{9}\left(\lambda_{0}+\epsilon_{0}^{2}\right)<\sqrt{C_{10}} / 4$, we infer from 63 that $\|p\|_{H^{2}}^{2} \leqslant \frac{1}{2} C_{10}$. This and 62 give $|a|^{2} \geqslant \frac{1}{2} C_{10}$.

Returning to 60, and using the above, we have

$$
\begin{aligned}
|\lambda| \sqrt{C_{10} / 2} & \leqslant C_{8} \epsilon^{2}\|y\|_{H^{2}}+|\lambda|\|\sigma\|_{\infty}\|p\|_{L^{2}}+\epsilon^{2} C_{8}\|p\|_{H^{2}} \\
& \leqslant C_{8} \epsilon^{2}\|y\|_{H^{2}}+\left(|\lambda|\|\sigma\|_{\infty}+C_{8} \epsilon^{2}\right) 2 C_{9}\left(|\lambda|+\epsilon^{2}\right) \\
& \leqslant C_{11}\left(\epsilon^{2}+\left(|\lambda|+\epsilon^{2}\right)^{2}\right)=C_{11}\left(\epsilon^{2}+|\lambda|^{2}+\epsilon^{4}+2|\lambda| \epsilon^{2}\right) .
\end{aligned}
$$

Choosing even smaller $\lambda_{0}$ such that $C_{11} \lambda_{0}<\frac{1}{2} \sqrt{C_{10} / 2}$, we get

$$
|\lambda| \leqslant C_{12}\left(\epsilon^{2}+\epsilon^{4}+2|\lambda| \epsilon^{2}\right) \leqslant C_{12}\left(2 \epsilon^{2}+2|\lambda| \epsilon^{2}\right) \leqslant C_{13} \epsilon^{2} .
$$

Finally, utilizing the estimate in 63 , we obtain $\|p\|_{H^{2}} \leqslant C_{14} \epsilon^{2}$.

This completes the proof of Proposition 1 with $M_{0}=\max \left\{C_{13}, C_{14}\right\}, a_{0}=\sqrt{C_{10} / 2}$.

\subsection{Existence, uniqueness, and simplicity of critical eigenvalues}

Following Nishiura [17], we call the eigenvalues $\lambda_{\epsilon}$ with the property $\lim _{\epsilon \rightarrow 0} \lambda_{\epsilon}=0$ critical.

PROPOSITION 2 The eigenvalue problem $\sqrt{36}-(\sqrt{38})$ has the following property: There exists $\epsilon_{1}<\epsilon_{0}$ such that for $0<\epsilon<\epsilon_{1}$, there is a critical eigenvalue $\lambda(\epsilon)$ satisfying $|\lambda(\epsilon)|=O\left(\epsilon^{2}\right)$. Furthermore $\lambda(\epsilon)$ is simple and it is the unique eigenvalue in the interval $\left[-\lambda_{0}, \lambda_{0}\right]$, where $\epsilon_{0}$ and $\lambda_{0}$ are as in Proposition 1

Proof

I. Existence of a critical eigenvalue. With $y$ as in Prop.1, we search for a solution of 444-466 of the form $h_{2}=y+p, p \perp y$. Substituting in (45) gives (recall that $-y^{\prime \prime}+q y=0$ )

$$
-p^{\prime \prime}+q p=\lambda \sigma y-\epsilon^{2} \tilde{K}_{2}^{\epsilon}(\lambda) y+\lambda \sigma p-\epsilon^{2} \tilde{K}_{2}^{\epsilon}(\lambda) p .
$$

Next, we will define a map $T:(\lambda, p) \mapsto(\hat{\lambda}, \hat{p})$ for

$$
\left.(\lambda, p) \in S=\left\{(\lambda, p) \in \mathbb{R} \times H^{2}:|\lambda| \leqslant M_{1} \epsilon^{2},\|p\|_{H^{2}} \leqslant M_{2} \epsilon^{2}, p \perp y \text { (in } L^{2}\right)\right\}
$$

( $M_{i}$ constants independent of $\epsilon$ and to be determined later). $S$ is a closed subset of the Banach space $\mathbb{R} \times H^{2}$ equipped with the norm $\|(\lambda, p)\|=|\lambda|+\|p\|_{H^{2}}$. We require that

$$
\begin{aligned}
-\hat{p}^{\prime \prime}+q \hat{p} & =\hat{\lambda} \sigma y-\epsilon^{2} \tilde{K}_{2}^{\epsilon}(\lambda) y+\lambda \sigma p-\epsilon^{2} \tilde{K}_{2}^{\epsilon}(\lambda) p, \\
\hat{\lambda} & =\frac{\left(\epsilon^{2} \tilde{K}_{2}^{\epsilon}(\lambda) y-\lambda \sigma p+\epsilon^{2} \tilde{K}_{2}^{\epsilon}(\lambda) p, y\right)}{(\sigma y, y)} .
\end{aligned}
$$


Notice that 66 implies that the right side of 65 is $L^{2}$-orthogonal to $y$. So for $(\lambda, p) \in S$, there is a unique $(\hat{\lambda}, \hat{p}) \in \mathbb{R} \times H^{2}, \hat{p} \perp y$, satisfying $(65)$; hence the map $T$ is well defined. Observe that if $(\lambda, p) \in S$ is a fixed point of $T$, then $\lambda$ and $h_{2}=y+p \neq 0$ (since $p \perp y$ ) satisfy (45).

Using (58) and the Cauchy-Schwarz inequality (recall $(\sigma y, y) \geqslant 1$ and $\|y\|_{L^{2}}=1$ ), we obtain $|\hat{\lambda}| \leqslant \epsilon^{2} C_{8}\|y\|_{H^{2}}+|\lambda|\|\sigma\|_{\infty}\|p\|_{L^{2}}+\epsilon^{2} C_{8}\|p\|_{H^{2}} \leqslant \epsilon^{2}\left[C_{8}\|y\|_{H^{2}}+\left(M_{1} M_{2}\|\sigma\|_{\infty}+M_{2} C_{8}\right) \epsilon^{2}\right]$.

Choosing

$$
\begin{aligned}
M_{1} & >\max \left\{C_{8}\|y\|_{H^{2}}+1, M_{0}\right\}, \\
M_{2} & >\max \left\{C_{7}\left(M_{1}\|\sigma\|_{\infty}+C_{8}\|y\|_{H^{2}}+1\right), M_{0} / a_{0}\right\}, \\
0<\epsilon_{1} & <\min \left\{\left(M_{1} M_{2}\|\sigma\|_{\infty}+C_{8} M_{2}\right)^{-1 / 2}, \epsilon_{0}, \sqrt{\lambda_{0} / M_{1}}\right\},
\end{aligned}
$$

we deduce that $|\hat{\lambda}| \leqslant M_{1} \epsilon^{2}$.

Also by Lemma 2 and (58),

$$
\begin{aligned}
\|\hat{p}\|_{H^{2}} & \leqslant C_{7}\left\|\hat{\lambda} \sigma y-\epsilon^{2} \tilde{K}_{2}^{\epsilon}(\lambda) y+\lambda \sigma p-\epsilon^{2} \tilde{K}_{2}^{\epsilon}(\lambda) p\right\|_{L^{2}} \\
& \leqslant C_{7}\left(M_{1} \epsilon^{2}\|\sigma\|_{\infty}+\epsilon^{2} C_{8}\|y\|_{H^{2}}+M_{1} \epsilon^{2}\|\sigma\|_{\infty} M_{2} \epsilon^{2}+\epsilon^{2} C_{8} M_{2} \epsilon^{2}\right) \\
& \leqslant C_{7} \epsilon^{2}\left(M_{1}\|\sigma\|_{\infty}+C_{8}\|y\|_{H^{2}}+1\right) \quad(\text { by (69)) } \\
& \left.\leqslant M_{2} \epsilon^{2} \quad \text { (by (68) }\right) .
\end{aligned}
$$

Hence $T: S \rightarrow S$.

The next step is to show that $T$ is a contraction on the closed set $S$. Let $\left(\lambda_{1}, p_{1}\right),\left(\lambda_{2}, p_{2}\right) \in S$; then

$\left|\hat{\lambda}_{1}-\hat{\lambda}_{2}\right| \leqslant \epsilon^{2}\left(\left\|\tilde{K}_{2}^{\epsilon}\left(\lambda_{1}\right) y-\tilde{K}_{2}^{\epsilon}\left(\lambda_{2}\right) y\right\|_{L^{2}}+\left\|\tilde{K}_{2}^{\epsilon}\left(\lambda_{1}\right) p_{1}-\tilde{K}_{2}^{\epsilon}\left(\lambda_{2}\right) p_{2}\right\|_{L^{2}}\right)+\|\sigma\|_{\infty}\left\|\lambda_{1} p_{1}-\lambda_{2} p_{2}\right\|_{L^{2}}$.

Using (58) and 59] gives

$$
\begin{aligned}
\left\|\tilde{K}_{2}^{\epsilon}\left(\lambda_{1}\right) p_{1}-\tilde{K}_{2}^{\epsilon}\left(\lambda_{2}\right) p_{2}\right\|_{L^{2}} & \leqslant\left\|\tilde{K}_{2}^{\epsilon}\left(\lambda_{1}\right)\left(p_{1}-p_{2}\right)\right\|_{L^{2}}++\left\|\tilde{K}_{2}^{\epsilon}\left(\lambda_{1}\right) p_{2}-\tilde{K}_{2}^{\epsilon}\left(\lambda_{2}\right) p_{2}\right\|_{L^{2}} \\
& \leqslant C_{8}\left\|p_{1}-p_{2}\right\|_{H^{2}}+C_{8}\left|\lambda_{1}-\lambda_{2}\right|\left\|p_{2}\right\|_{H^{2}} \\
& \leqslant C_{8}\left\|p_{1}-p_{2}\right\|_{H^{2}}+C_{8} M_{2} \epsilon^{2}\left|\lambda_{1}-\lambda_{2}\right| .
\end{aligned}
$$

Also

$$
\begin{aligned}
\left\|\lambda_{1} p_{1}-\lambda_{2} p_{2}\right\|_{L^{2}} & \leqslant\left|\lambda_{1}\right|\left\|p_{1}-p_{2}\right\|_{L^{2}}+\left|\lambda_{1}-\lambda_{2}\right|\left\|p_{2}\right\|_{L^{2}} \\
& \leqslant M_{1} \epsilon^{2}\left\|p_{1}-p_{2}\right\|_{L^{2}}+M_{2} \epsilon^{2}\left|\lambda_{1}-\lambda_{2}\right| .
\end{aligned}
$$

Hence

$$
\left|\hat{\lambda}_{1}-\hat{\lambda}_{2}\right|=O\left(\epsilon^{2}\right)\left(\left|\lambda_{1}-\lambda_{2}\right|+\left\|p_{1}-p_{2}\right\|_{H^{2}}\right) .
$$

Moreover,

$$
\begin{aligned}
-\left(\hat{p}_{1}-\hat{p}_{2}\right)^{\prime \prime}+q\left(\hat{p}_{1}-\hat{p}_{2}\right)= & \left(\hat{\lambda}_{1}-\hat{\lambda}_{2}\right) \sigma y-\epsilon^{2}\left(\tilde{K}_{2}^{\epsilon}\left(\lambda_{1}\right) y-\tilde{K}_{2}^{\epsilon}\left(\lambda_{2}\right) y\right) \\
& +\sigma\left(\lambda_{1} p_{1}-\lambda_{2} p_{2}\right)-\epsilon^{2}\left(\tilde{K}_{2}^{\epsilon}\left(\lambda_{1}\right) p_{1}-\tilde{K}_{2}^{\epsilon}\left(\lambda_{2}\right) p_{2}\right) .
\end{aligned}
$$

By Lemma2.

$$
\begin{aligned}
\left\|\hat{p}_{1}-\hat{p}_{2}\right\|_{H^{2}} \leqslant & C_{7} \|\left(\hat{\lambda}_{1}-\hat{\lambda}_{2}\right) \sigma y-\epsilon^{2}\left(\tilde{K}_{2}^{\epsilon}\left(\lambda_{1}\right) y-\tilde{K}_{2}^{\epsilon}\left(\lambda_{2}\right) y\right) \\
& +\sigma\left(\lambda_{1} p_{1}-\lambda_{2} p_{2}\right)-\epsilon^{2}\left(\tilde{K}_{2}^{\epsilon}\left(\lambda_{1}\right) p_{1}-\tilde{K}_{2}^{\epsilon}\left(\lambda_{2}\right) p_{2}\right) \|_{L^{2}} .
\end{aligned}
$$


Working as before and using $(70)$, we get

$$
\left\|\hat{p}_{1}-\hat{p}_{2}\right\|_{H^{2}}=O\left(\epsilon^{2}\right)\left(\left|\lambda_{1}-\lambda_{2}\right|+\left\|p_{1}-p_{2}\right\|_{H^{2}}\right) .
$$

So combining the inequality above with (70) gives

$$
\left|\hat{\lambda}_{1}-\hat{\lambda}_{2}\right|+\left\|\hat{p}_{1}-\hat{p}_{2}\right\|_{H^{2}}=O\left(\epsilon^{2}\right)\left(\left|\lambda_{1}-\lambda_{2}\right|+\left\|p_{1}-p_{2}\right\|_{H^{2}}\right) .
$$

Choosing $\epsilon_{1}>0$ sufficiently small, we see that if $\epsilon<\epsilon_{1}$, then $T$ is a contraction. Applying the Banach fixed point theorem, we infer that there exists a unique $(\lambda, p) \in S$ such that $T(\lambda, p)=$ $(\lambda, p)$, that is, $\hat{\lambda}=\lambda$ and $\hat{p}=p$. So by 65, and 66, we get

$$
-p^{\prime \prime}+q p=\lambda \sigma y-\epsilon^{2} \tilde{K}_{2}^{\epsilon}(\lambda) y+\lambda \sigma p-\epsilon^{2} \tilde{K}_{2}^{\epsilon}(\lambda) p .
$$

Obviously $\lambda,\left(h_{1}, h_{2}\right) \neq 0$ with $h_{1}=K_{1}^{\epsilon}(\lambda) h_{2}$ satisfy $(44)$ and $(45)$ and we can assume that 446 is also satisfied. Hence we found an eigenvalue $\lambda$ with $|\lambda| \leqslant M_{1} \epsilon^{2}$ corresponding to an eigenfunction $\left(h_{1}, h_{2}\right)$.

II. Uniqueness and simplicity of the critical eigenvalue. Suppose that for some $\epsilon<\epsilon_{1}$ we have an eigenvalue $\bar{\lambda}$ (not necessarily $\bar{\lambda} \neq \lambda$ ) such that $|\bar{\lambda}| \leqslant \lambda_{0}$. Then by Proposition 1 .

$$
|\bar{\lambda}| \leqslant M_{0} \epsilon^{2}, \quad\|\bar{p}\|_{H^{2}} \leqslant M_{0} \epsilon^{2}, \quad|\bar{a}| \geqslant a_{0},
$$

where $\bar{h}_{2}=\bar{a} y+\bar{p}, y \perp \bar{p}$ (again we suppress the dependence of $\lambda, \bar{\lambda}, \bar{h}_{2}, \bar{p}, \bar{a}$ on $\epsilon$ ) with $\left(\bar{h}_{1}, \bar{h}_{2}\right)$ a normalized eigenvector. Using (67)-669, we deduce from the above that

$$
|\bar{\lambda}| \leqslant M_{1} \epsilon^{2}, \quad\|\bar{p} / \bar{a}\|_{H^{2}} \leqslant M_{2} \epsilon^{2},
$$

thus $(\bar{\lambda}, \bar{p} / \bar{a})$ is a fixed point of $T$ in $S$. Hence $\bar{\lambda}=\lambda$ and $\bar{p} / \bar{a}=p$. That is, $\bar{h}_{2}=\bar{a}(y+p)=\tilde{a} h_{2}$ and

$$
\bar{h}_{1}=K_{1}^{\epsilon}(\bar{\lambda}) \bar{h}_{2}=\tilde{a} K_{1}^{\epsilon}(\lambda) h_{2}=\tilde{a} h_{1},
$$

where $\tilde{a}$ depends on $\epsilon>0$ and is due to the normalization of $\left(h_{1}, h_{2}\right)$. Since $\left(h_{1}, h_{2}\right)$ and $\left(\bar{h}_{1}, \bar{h}_{2}\right)$ are normalized, we conclude that $|\tilde{a}|=1$. Hence if $\epsilon<\epsilon_{1}$, then $\lambda$ is the unique eigenvalue in $\left[-\lambda_{0}, \lambda_{0}\right]$ and it is simple. The proof of Proposition 2 is complete.

\subsection{The lower bound of the spectrum}

LEMma 3 Let $\lambda<0$ be an eigenvalue with corresponding $L^{2}$ normalized eigenfunction $\left(h_{1}, h_{2}\right)$. Then $\left\|h_{2}\right\|_{H^{2}} \leqslant C_{16}$, where $C_{16}$ is independent of $\lambda, \epsilon$.

Proof. Let $\lambda<0$ be an eigenvalue of (36)-(38) with corresponding eigenfunction $\left(h_{1}, h_{2}\right)$. From (37),

$$
\begin{aligned}
\left(h_{2}^{\prime \prime}+\lambda h_{2}\right)^{2} & =\left(g_{x r} h_{1}+g_{r r} h_{2}\right)^{2} \leqslant\left(g_{x r}^{2}+g_{r r}^{2}\right)\left(h_{1}^{2}+h_{2}^{2}\right) \\
& \leqslant \sup _{s} g_{x r}^{2}+g_{r r}^{2}\left(h_{1}^{2}+h_{2}^{2}\right) .
\end{aligned}
$$

Let $C_{15}=\sup _{s}\left(g_{x r}^{2}+g_{r r}^{2}\right)$. Then via 38 and integration by parts, we have

$$
\int_{-\infty}^{\infty}\left(h_{2}^{\prime \prime}\right)^{2} \mathrm{~d} s+\lambda^{2} \int_{-\infty}^{\infty} h_{2}^{2} \mathrm{~d} s-2 \lambda \int_{-\infty}^{\infty}\left(h_{2}^{\prime}\right)^{2} \mathrm{~d} s \leqslant C_{15} .
$$


Since $\lambda<0$, from the inequality above we obtain

$$
\int_{-\infty}^{\infty}\left(h_{2}^{\prime \prime}\right)^{2} \mathrm{~d} s \leqslant C_{15}, \quad \text { and so } \quad \int_{-\infty}^{\infty}\left(h_{2}^{\prime \prime}\right)^{2} \mathrm{~d} s+\int_{-\infty}^{\infty} h_{2}^{2} \mathrm{~d} s \leqslant C_{15}+1 .
$$

Also from the identity $-2 h_{2} h_{2}^{\prime \prime} \leqslant\left(h_{2}^{\prime \prime}\right)^{2}+h_{2}^{2}$, we get

$$
2 \int_{-\infty}^{\infty}\left(h_{2}^{\prime}\right)^{2} \mathrm{~d} s \leqslant C_{15}+1
$$

Hence $\left\|h_{2}\right\|_{H^{2}} \leqslant \sqrt{\frac{3}{2}\left(C_{15}+1\right)}=C_{16}$. The proof of Lemma 3 is complete.

Proposition 3 Let $\epsilon_{1}, \lambda_{0}$ be as in Proposition 2. There is $0<\epsilon_{2}<\epsilon_{1}$ such that for $0<\epsilon<\epsilon_{2}$, there are no eigenvalues $\lambda$ with $\lambda \leqslant-\lambda_{0}$.

Proof. Suppose that there is an eigenvalue $\lambda$ with $\lambda \leqslant-\lambda_{0}$. By 45 ,

$$
-h_{2}^{\prime \prime}+q h_{2}=\lambda\left[1+\frac{g_{x r}^{2}}{g_{x x}\left(g_{x x}-\lambda\right)}\right] h_{2}-\epsilon^{2} g_{x r} K_{2}^{\epsilon}(\lambda) h_{2} .
$$

We know that $\int_{-\infty}^{\infty}\left[\left(h^{\prime}\right)^{2}+q h^{2}\right] \mathrm{d} s \geqslant 0$ for all $h \in H^{2}$, since the spectrum of the bistable operator $B h=-h^{\prime \prime}+q h$ is positive. Also we recall that $\lambda \leqslant-\lambda_{0}$. Multiplying both sides of the above equation by $h_{2}$ and integrating by parts gives

$$
\begin{aligned}
\lambda_{0}\left\|h_{2}\right\|_{L^{2}}^{2} & \leqslant \epsilon^{2}\left|\left(g_{x r} K_{2}^{\epsilon}(\lambda) h_{2}, h_{2}\right)\right| \leqslant \epsilon^{2} \sup _{s}\left|g_{x r}\right|\left\|K_{2}^{\epsilon}(\lambda) h_{2}\right\|_{L^{2}}\left\|h_{2}\right\|_{L^{2}} \\
& \leqslant \epsilon^{2} \sup _{s}\left|g_{x r}\right|\left\|K_{2}^{\epsilon}(\lambda) h_{2}\right\|_{L^{2}} \leqslant \epsilon^{2} \sup _{s}\left|g_{x r}\right| C_{8}\left\|h_{2}\right\|_{H^{2}},
\end{aligned}
$$

where we used the estimate $\left\|h_{2}\right\|_{L^{2}} \leqslant 1$ and 48 . Now using Lemma 3 , we get $\left\|h_{2}\right\|_{L^{2}} \leqslant C_{17} \epsilon^{2}$. Moreover, by [47],

$$
\left\|h_{1}\right\|_{L^{2}}=\left\|K_{1}^{\epsilon}(\lambda) h_{2}\right\|_{L^{2}} \leqslant C_{4}\left\|h_{2}\right\|_{L^{2}} \leqslant C_{18} \epsilon^{2} .
$$

Obviously if $\epsilon<\epsilon_{2}$, then these estimates contradict the equality $\left\|h_{1}\right\|_{L^{2}}^{2}+\left\|h_{2}\right\|_{L^{2}}^{2}=1$. The proof of Proposition 3 is complete.

\subsection{The basic result on the spectrum of $L_{\epsilon}$}

THEOREM 1 If $\epsilon>0$ is sufficiently small, then the spectrum of $L_{\epsilon}$ (cf. (27) ) has the following form:

(i) At the bottom of the spectrum, there is a simple eigenvalue $\lambda_{1}(\epsilon)=O\left(\epsilon^{2}\right)$. Furthermore the $L^{2} \times L^{2}$ normalized eigenfunction $\left(h_{1}^{\epsilon}, h_{2}^{\epsilon}\right)$ corresponding to $\lambda_{1}(\epsilon)$ satisfies

$$
\begin{gathered}
\left.h_{2}^{\epsilon}=a_{\epsilon} \frac{r_{0}^{\prime}}{\left\|r_{0}^{\prime}\right\|_{L^{2}}}+p_{\epsilon}, \quad p_{\epsilon} \perp r_{0}^{\prime} \quad \text { (in } L^{2}\right), \\
a_{\epsilon} \in \mathbb{R}, \quad p_{\epsilon} \in H^{2}, \quad\left|a_{\epsilon}\right| \geqslant a_{0}>0, \quad\left\|p_{\epsilon}\right\|_{H^{2}}=O\left(\epsilon^{2}\right) .
\end{gathered}
$$

(ii) The rest of the spectrum is contained in $\left[\lambda_{0}, \infty\right)$, where $a_{0}, \lambda_{0}$ are positive constants independent of $\epsilon$. 
Proof. By Proposition 2 if $\epsilon<\epsilon_{2}$, there exists a unique eigenvalue $\lambda(\epsilon) \in\left[-\lambda_{0}, \lambda_{0}\right]$. Moreover $\lambda(\epsilon)=O\left(\epsilon^{2}\right)$ and $\lambda$ is simple. Also the corresponding $h_{2}$ satisfies the required estimate. Furthermore, by Proposition 3 there is no eigenvalue in $\left(-\infty,-\lambda_{0}\right]$. Hence $\lambda$ is the only eigenvalue in $\left(-\infty, \lambda_{0}\right]$ and consequently it is the principal eigenvalue. Denote it by $\lambda_{1}$. By the results in Section 2.1. the essential spectrum of $L_{\epsilon}$ is contained in $\left[C_{3}, \infty\right)$, where $C_{3}>0$ is independent of $\epsilon$, and $\lambda_{0}<C_{3}$.

The proof of Theorem 1 is complete.

\section{The heteroclinic}

We seek a solution $u=(x, r)$ of (12) of the form $u=u_{0}+\tilde{u}$, where $u_{0}=\left(x_{0}, r_{0}\right)$ and $\tilde{u}=(\tilde{x}, \tilde{r}) \in$ $H^{2} \times H^{2}, \tilde{u} \perp u_{0}^{\prime}$ in $L^{2}$. Obviously, $u$ has the required behavior 14 at infinity. It remains to be shown that $u$ satisfies 12 , from which we also infer that $u \in C^{2} \times C^{2}$.

We have

$$
L_{\epsilon} \tilde{u}=N(\tilde{u})+E,
$$

where

$$
N(\tilde{u})=-\left(\begin{array}{l}
g_{x}\left(\tilde{x}+x_{0}, \tilde{r}+r_{0}\right)-g_{x}\left(x_{0}, r_{0}\right)-g_{x x}\left(x_{0}, r_{0}\right) \tilde{x}-g_{x r}\left(x_{0}, r_{0}\right) \tilde{r} \\
g_{r}\left(\tilde{x}+x_{0}, \tilde{r}+r_{0}\right)-g_{r}\left(x_{0}, r_{0}\right)-g_{x r}\left(x_{0}, r_{0}\right) \tilde{x}-g_{r r}\left(x_{0}, r_{0}\right) \tilde{r}
\end{array}\right)
$$

and

$$
E=\epsilon^{2}\left(\begin{array}{c}
x_{0}^{\prime \prime} \\
0
\end{array}\right) .
$$

The existence of a solution $\tilde{u}$ of 71 follows from the following propositions (see Theorem 2 in Section 3.1 below).

Proposition 4 If $\epsilon$ is sufficiently small, there exists a pair $(c, \tilde{u})$ with $c \in \mathbb{R}$ and $\tilde{u} \in H^{2} \times$ $H^{2}, \tilde{u} \perp u_{0}^{\prime}$, such that

$$
L \tilde{u}=-c u_{0}^{\prime}+N(\tilde{u})+E, \quad\|\tilde{u}\|_{1, \epsilon}=O\left(\epsilon^{2}\right),
$$

where $\|(x, r)\|_{1, \epsilon}^{2}=\epsilon^{2}\left\|x^{\prime}\right\|_{L^{2}}^{2}+\left\|r^{\prime}\right\|_{L^{2}}^{2}+\|x\|_{L^{2}}^{2}+\|r\|_{L^{2}}^{2}$.

Proposition 5 Let $(c, \tilde{u})$ be as in Proposition 4 . Then $c=0$.

In the remainder of the paper, it is assumed that $0<\epsilon<\epsilon_{2}$.

Lemma 4 For $\epsilon$ sufficiently small, we have

(i) $\left\|\boldsymbol{h}-\left(a /\left\|r_{0}^{\prime}\right\|_{L^{2}}\right) u_{0}^{\prime}\right\|_{L^{2} \times L^{2}} \leqslant C_{21} \epsilon^{2}$,

(ii) $\left|\left(\boldsymbol{h}, u_{0}^{\prime}\right)\right| \geqslant \frac{1}{2}\left\|r_{0}^{\prime}\right\|_{L^{2}}$,

where $\boldsymbol{h}=\left(h_{1}, h_{2}\right)$ is the normalized eigenfunction corresponding to $\lambda_{1}$ (recall $h_{2}=a y+p, y=$ $\left.r_{0}^{\prime} /\left\|r_{0}^{\prime}\right\|_{L^{2}}\right)$ and $u_{0}=\left(x_{0}, r_{0}\right) . C_{21}$ is independent of $\epsilon$ (recall that $\lambda_{1}, \boldsymbol{h}, \boldsymbol{a}$ depend on $\left.\epsilon\right)$. 
Proof. For (i), we have

$$
\begin{aligned}
\left\|\boldsymbol{h}-\frac{a}{\left\|r_{0}^{\prime}\right\|_{L^{2}}} u_{0}^{\prime}\right\|_{L^{2} \times L^{2}} & \leqslant\left\|h_{1}-\frac{a}{\left\|r_{0}^{\prime}\right\|_{L^{2}}} x_{0}^{\prime}\right\|_{L^{2}}+\left\|h_{2}-\frac{a}{\left\|r_{0}^{\prime}\right\|_{L^{2}}} r_{0}^{\prime}\right\|_{L^{2}} \\
& =\left\|K_{1}^{\epsilon}\left(\lambda_{1}\right)(a y+p)-\frac{a}{\left\|r_{0}^{\prime}\right\|_{L^{2}}} x_{0}^{\prime}\right\|_{L^{2}}+\left\|h_{2}-a y\right\|_{L^{2}} \\
& \leqslant\left\|K_{1}^{\epsilon}\left(\lambda_{1}\right) p\right\|_{L^{2}}+\frac{|a|}{\left\|r_{0}^{\prime}\right\|_{L^{2}}}\left\|K_{1}^{\epsilon}\left(\lambda_{1}\right) r_{0}^{\prime}-x_{0}^{\prime}\right\|_{L^{2}}+\|p\|_{L^{2}} .
\end{aligned}
$$

By 477 , the estimates $\|p\|_{H^{2}}=O\left(\epsilon^{2}\right),|a| \leqslant 1$ and the definition of $K_{2}^{\epsilon}(\lambda)$, this is

$$
\begin{aligned}
& \leqslant C_{19} \epsilon^{2}+C_{20}\left\|-\frac{g_{x r}}{g_{x x}-\lambda_{1}} r_{0}^{\prime}+\epsilon^{2} K_{2}^{\epsilon}\left(\lambda_{1}\right) r_{0}^{\prime}+\frac{g_{x r}}{g_{x x}} r_{0}^{\prime}\right\|_{L^{2}} \\
& \leqslant C_{19} \epsilon^{2}+C_{20}\left\|\frac{\lambda_{1} g_{x r} r_{0}^{\prime}}{g_{x x}\left(g_{x x}-\lambda_{1}\right)}\right\|_{L^{2}}+\epsilon^{2} C_{20}\left\|K_{2}^{\epsilon}\left(\lambda_{1}\right) r_{0}^{\prime}\right\|_{L^{2}} \leqslant C_{21} \epsilon^{2},
\end{aligned}
$$

where we used the fact that $\left|\lambda_{1}\right|=O\left(\epsilon^{2}\right)$ and Lemma 1 .

(ii) follows trivially from (i) via $\|\boldsymbol{h}\|_{L^{2} \times L^{2}}=1$.

Lemma 5 For $\epsilon$ sufficiently small and every $w$ such that $w \in H^{2} \times H^{2}, w \perp \boldsymbol{h}$, the following estimates hold:

(i) $\left(L_{\epsilon} w, w\right) \geqslant \lambda_{0}\|w\|_{L^{2}}^{2}$,

(ii) $\left(L_{\epsilon} w, w\right) \geqslant C_{23}\|w\|_{1, \epsilon}^{2}$,

where $\lambda_{0}$ is as in Proposition 1 and $C_{23}>0$ is independent of $\epsilon$ and $w$.

Proof. (i) is obvious from the variational characterization of the eigenvalues of self-adjoint operators.

(ii) Let $w=\left(w_{1}, w_{2}\right) \in H^{2} \times H^{2}, w \perp \boldsymbol{h}$, and $0<C<1$. Then

$$
\begin{aligned}
\left(L_{\epsilon} w, w\right)-C\|w\|_{1, \epsilon}^{2} & =(1-C)\left(L_{\epsilon} w, w\right)+C\left[\left(L_{\epsilon} w, w\right)-\|w\|_{1, \epsilon}^{2}\right] \\
& \geqslant(1-C) \lambda_{0}\|w\|_{L^{2}}^{2}+C \int_{-\infty}^{\infty}\left[\left(g_{x x}-1\right) w_{1}^{2}+2 g_{x r} w_{1} w_{2}+\left(g_{r r}-1\right) w_{2}^{2}\right] \mathrm{d} s \\
& \geqslant(1-C) \lambda_{0}\|w\|_{L^{2}}^{2}-C C_{22}\|w\|_{L^{2}}^{2} .
\end{aligned}
$$

Choosing $C$ sufficiently small completes the proof with $C_{23}:=C$.

Lemma 6 Let $U_{i} \in H^{1} \times H^{1},\left\|U_{i}\right\|_{1, \epsilon} \leqslant M \epsilon^{2}, i=1,2, M>1, \epsilon<1$. Then $\left\|N\left(U_{i}\right)\right\|_{L^{2} \times L^{2}} \leqslant$ $C_{24} M^{3} \epsilon^{3}$ and

$$
\left\|N\left(U_{1}\right)-N\left(U_{2}\right)\right\|_{L^{2} \times L^{2}} \leqslant C_{24} M^{2} \epsilon^{\delta}\left\|U_{1}-U_{2}\right\|_{L^{2} \times L^{2}},
$$

where $C_{24}, \delta>0$ are independent of $\epsilon, M, U_{i}$.

Proof. Let $U=(x, r) \in H^{1} \times H^{1}$ with $\|U\|_{1, \epsilon} \leqslant M \epsilon^{2}$. Then $\|x\|_{H^{1}} \leqslant M \epsilon,\|r\|_{H^{1}} \leqslant$ $M \epsilon^{2},\|x\|_{L^{2}} \leqslant M \epsilon^{2},\|r\|_{L^{2}} \leqslant M \epsilon^{2}$. From 16, , 17), (72),

$$
N(U)=\left(\begin{array}{c}
6 r^{2}-2 x r^{2}-4 x r r_{0}-2 x_{0} r^{2}-4 x^{3}-12 x^{2} x_{0} \\
12 x r-2 x^{2} r-2 x^{2} r_{0}-4 x x_{0} r-3 r^{3}-9 r^{2} r_{0}
\end{array}\right) .
$$


We recall the Gagliardo-Nirenberg inequality [12]: if $u \in H^{1}$, then $\|u\|_{L^{p}} \leqslant C\|u\|_{H^{1}}^{\theta}\|u\|_{L^{2}}^{1-\theta}$ for $p \geqslant 2,0 \leqslant \theta \leqslant 1, \theta \geqslant 1 / 2-1 / p$. We estimate a typical term to explain the procedure.

Consider the term $x r^{2}$ in the expression of $N(U)$ :

$$
\left\|x r^{2}\right\|_{L^{2}} \leqslant\left\|x^{2}\right\|_{L^{2}}^{1 / 2}\left\|r^{4}\right\|_{L^{2}}^{1 / 2}=\|x\|_{L^{4}}\|r\|_{L^{8}}^{2} \leqslant C\|x\|_{H^{1}}^{1 / 4}\|x\|_{L^{2}}^{3 / 4}\|r\|_{H^{1}}^{3 / 4}\|r\|_{L^{2}}^{5 / 4} \leqslant C M^{3} \epsilon^{23 / 4} .
$$

The rest of the terms can be estimated analogously. Let $U_{i}=\left(x_{i}, r_{i}\right) \in H^{1} \times H^{1}$ be such that $\left\|U_{i}\right\|_{1, \epsilon} \leqslant M \epsilon^{2}, i=1,2$. Then $\left\|x_{i}\right\|_{\infty} \leqslant C M \epsilon^{3 / 2}$ and $\left\|r_{i}\right\|_{\infty} \leqslant C M \epsilon^{2}$. We will treat a typical term in $N\left(U_{1}\right)-N\left(U_{2}\right)$ and leave the rest to the reader:

$$
\begin{aligned}
\left\|x_{2} r_{2}^{2}-x_{1} r_{1}^{2}\right\|_{L^{2}} & \leqslant\left\|x_{2} r_{2}^{2}-x_{2} r_{1}^{2}\right\|_{L^{2}}+\left\|x_{2} r_{1}^{2}-x_{1} r_{1}^{2}\right\|_{L^{2}} \\
& \leqslant\left\|x_{2}\right\|_{\infty}\left\|\left(-r_{2}+r_{1}\right)\left(-r_{2}-r_{1}\right)\right\|_{L^{2}}+\left\|r_{1}^{2}\right\|_{\infty}\left\|x_{2}-x_{1}\right\|_{L^{2}} \\
& \leqslant 2 M^{2} C^{2} \epsilon^{3}\left\|r_{2}-r_{1}\right\|_{L^{2}}+C^{2} M^{2} \epsilon^{4}\left\|x_{2}-x_{1}\right\|_{L^{2}} .
\end{aligned}
$$

\section{Proof of Proposition 4}

I. The transformation $\tilde{u} \mapsto w$. For $\tilde{u} \in L^{2} \times L^{2}, \tilde{u} \perp u_{0}^{\prime}$, set

$$
w=\tilde{u}-\left(\tilde{u}, h-\frac{a}{\left\|r_{0}^{\prime}\right\|_{L^{2}}} u_{0}^{\prime}\right) h .
$$

By Lemma 4 (i), the right hand side is a small perturbation of the identity. Hence if $\epsilon$ is sufficiently small, the above linear transformation is invertible and $\tilde{u}=Q(w)$. Also note that we have the equivalence $w \perp h \Leftrightarrow \tilde{u} \perp u_{0}^{\prime}$. Furthermore, if $\epsilon$ is sufficiently small, then

$$
\left\|Q\left(w_{1}\right)-Q\left(w_{2}\right)\right\|_{L^{2} \times L^{2}} \leqslant C_{25}\left\|w_{1}-w_{2}\right\|_{L^{2} \times L^{2}}
$$

and $\|Q(w)\|_{1, \epsilon} \leqslant C_{25}\|w\|_{1, \epsilon}$, for every $w_{1}, w_{2} \in L^{2} \times L^{2}, w \in H^{1} \times H^{1}, w_{1}, w_{2}, w \perp h$, where $C_{25}>1$ is a constant independent of $\epsilon, w, w_{1}, w_{2}$.

II. The w-equation. Substituting (74) into (73) gives

$$
L w=-c u_{0}^{\prime}+N(Q(w))+E-(Q(w), \boldsymbol{h}) \lambda_{1} \boldsymbol{h} .
$$

We define a function $C: H^{2} \times H^{2} \rightarrow \mathbb{R}$ by

$$
C(W)=\frac{1}{\left(u_{0}^{\prime}, \boldsymbol{h}\right)}\left(N(Q(W))+E-\lambda_{1} Q(W), \boldsymbol{h}\right)
$$

(from Lemma 4 (ii), $\left.\left|\left(u_{0}^{\prime}, \boldsymbol{h}\right)\right| \geqslant \frac{1}{2}\left\|r_{0}^{\prime}\right\|_{L^{2}}\right)$. We seek $W \in H^{2} \times H^{2}, W \perp \boldsymbol{h}$, such that

$$
L W=-C(W) u_{0}^{\prime}+N(Q(W))+E-(Q(W), \boldsymbol{h}) \lambda_{1} \boldsymbol{h} .
$$

We can define an operator

$$
T:\left(H^{2} \times H^{2}\right) \cap \boldsymbol{h}^{\perp} \rightarrow\left(H^{2} \times H^{2}\right) \cap \boldsymbol{h}^{\perp}
$$

by $T W=\hat{W}$, where $\hat{W}$ is the unique solution in $\left(H^{2} \times H^{2}\right) \cap \boldsymbol{h}^{\perp}$ of

$$
L \hat{W}=-C(W) u_{0}^{\prime}+N(Q(W))+E-(Q(W), \boldsymbol{h}) \lambda_{1} \boldsymbol{h} .
$$

This map is well defined via (76) and Lemma 5 (i). 
Let $S=\left\{W \in\left(H^{2} \times H^{2}\right) \cap \boldsymbol{h}^{\perp}:\|W\|_{1, \epsilon} \leqslant M_{3} \epsilon^{2}\right\}$, where $M_{3}>1$ is to be chosen. Let $W \in S$. Then

$$
\begin{aligned}
|C(W)| & \leqslant C_{26}\left\|N(Q(W))+E-\lambda_{1} Q(W)\right\|_{L^{2} \times L^{2}} \\
& \leqslant C_{26}\left(C_{24} C_{25}^{3} M_{3}^{3} \epsilon^{3}+\left\|x_{0}^{\prime \prime}\right\|_{L^{2}} \epsilon^{2}+M_{1} \epsilon^{2} C_{25} M_{3} \epsilon^{2}\right) .
\end{aligned}
$$

From (78) via Lemma 5(ii) and the above, we have

$$
\|T W\|_{1, \epsilon} \leqslant C_{27}\left(C_{24} C_{25}^{3} M_{3}^{3} \epsilon^{3}+\left\|x_{0}^{\prime \prime}\right\|_{L^{2}} \epsilon^{2}+M_{1} C_{25} M_{3} \epsilon^{4}\right) .
$$

By choosing $M_{3}$ sufficiently large and $\epsilon$ sufficiently small, we conclude that $\|T W\|_{1, \epsilon} \leqslant M_{3} \epsilon^{2}$, i.e. $T S \subset S$.

Now let $W_{1}, W_{2} \in S$; we have

$$
\begin{aligned}
\left|C\left(W_{1}\right)-C\left(W_{2}\right)\right| & \leqslant C_{26}\left\|N\left(Q\left(W_{1}\right)\right)-N\left(Q\left(W_{2}\right)\right)\right\|_{L^{2} \times L^{2}}+C_{26} M_{1} \epsilon^{2}\left\|Q\left(W_{1}\right)-Q\left(W_{2}\right)\right\|_{L^{2} \times L^{2}} \\
& \leqslant C_{26}\left(C_{24} C_{25}^{2} M_{3}^{2} \epsilon^{\delta}+M_{1} \epsilon^{2}\right)\left\|Q\left(W_{1}\right)-Q\left(W_{2}\right)\right\|_{L^{2} \times L^{2}} \\
& \leqslant C_{26}\left(C_{24} C_{25}^{2} M_{3}^{2} \epsilon^{\delta}+M_{1} \epsilon^{2}\right) C_{25}\left\|W_{1}-W_{2}\right\|_{1, \epsilon}
\end{aligned}
$$

and

$$
\left\|T W_{1}-T W_{2}\right\|_{1, \epsilon} \leqslant C_{28}\left(C_{24} C_{25}^{2} M_{3}^{2} \epsilon^{\delta}+M_{1} \epsilon^{2}\right) C_{25}\left\|W_{1}-W_{2}\right\|_{1, \epsilon} .
$$

If $\epsilon$ is sufficiently small, $T$ is a contraction on the closed set $S$ and $T S \subset S$. Thus there exists $W \in S$ such that $T W=W$. Hence we obtain a pair $C=C(W) \in \mathbb{R}$ and $W \in H^{2} \times H^{2}$, with $W \perp \boldsymbol{h}$ and $\|W\|_{1, \epsilon} \leqslant M_{3} \epsilon^{2}$, that satisfies $(75)$. Obviously $C=C(W)$ and $\tilde{u}=Q(W)$ satisfy 73 and $\tilde{u} \in H^{2} \times H^{2}, \tilde{u} \perp u_{0}^{\prime},\|\tilde{u}\|_{1, \epsilon} \leqslant C_{25} M_{3} \epsilon^{2}$.

This completes the proof of Proposition 4

REMARK 1 From 73 we see that $\tilde{u} \in C^{2} \times C^{2}$.

Proof of Proposition 5 Set $u=u_{0}+\tilde{u}=(x, r) \in C^{2} \times C^{2}$ (recall that $\tilde{u}$ depends on $\epsilon$ and $\left.\|\tilde{u}\|_{1, \epsilon}=O\left(\epsilon^{2}\right)\right)$. Then $x(s) \rightarrow 0$ and $r(s) \rightarrow 0$ as $s \rightarrow-\infty ; x(s) \rightarrow 1$ and $r(s) \rightarrow \sqrt{2}$ as $s \rightarrow \infty$; and $x^{\prime}(s), r^{\prime}(s) \rightarrow 0$ as $|s| \rightarrow \infty$. Rewriting $(73)$ in a more suitable form, we have

$$
\left(\begin{array}{c}
\epsilon^{2} x^{\prime \prime}-g_{x}(x, r) \\
r^{\prime \prime}-g_{r}(x, r)
\end{array}\right)=c u_{0}^{\prime} .
$$

Now taking the inner product of both sides with $u^{\prime}$ gives

$$
\begin{gathered}
\int_{-\infty}^{\infty}\left(\epsilon^{2} x^{\prime \prime} x^{\prime}+r^{\prime \prime} r^{\prime}-g_{x}(x, r) x^{\prime}-g_{r}(x, r) r^{\prime}\right) \mathrm{d} s=c\left(u_{0}^{\prime}, u_{0}^{\prime}+\tilde{u}^{\prime}\right), \\
\int_{-\infty}^{\infty} \frac{\mathrm{d}}{\mathrm{d} s}\left(\frac{\epsilon^{2}}{2}\left(x^{\prime}\right)^{2}+\frac{1}{2}\left(r^{\prime}\right)^{2}-g(x, r)\right) \mathrm{d} s=c\left(\left\|u_{0}^{\prime}\right\|_{L^{2} \times L^{2}}^{2}+\left(u_{0}^{\prime}, \tilde{u}^{\prime}\right)\right),
\end{gathered}
$$

i.e.

$$
c\left(\left\|u_{0}^{\prime}\right\|_{L^{2} \times L^{2}}^{2}+\left(u_{0}^{\prime}, \tilde{u}^{\prime}\right)\right)=g(0,0)-g(1, \sqrt{2})=0 .
$$

From this, we assert that for sufficiently small $\epsilon$ we have $c=0$. Indeed, since $\|\tilde{u}\|_{1, \epsilon} \leqslant C_{25} M_{3} \epsilon^{2}$, we have $\left\|\tilde{u}^{\prime}\right\|_{L^{2} \times L^{2}} \leqslant C_{25} M_{3} \epsilon$, and so

$$
\begin{aligned}
\left\|u_{0}^{\prime}\right\|_{L^{2} \times L^{2}}^{2}+\left(u_{0}^{\prime}, \tilde{u}^{\prime}\right) & \geqslant\left\|u_{0}^{\prime}\right\|_{L^{2} \times L^{2}}^{2}-\left\|u_{0}^{\prime}\right\|_{L^{2} \times L^{2}}\left\|\tilde{u}^{\prime}\right\|_{L^{2} \times L^{2}} \\
& \geqslant\left\|u_{0}^{\prime}\right\|_{L^{2} \times L^{2}}\left(\left\|u_{0}^{\prime}\right\|_{L^{2} \times L^{2}}-C_{25} M_{3} \epsilon\right)>0
\end{aligned}
$$

if $\epsilon$ is sufficiently small. This completes the proof of Proposition 5 


\subsection{The existence theorem}

We have therefore proved the following, which is the same as Theorem A:

THEOREM 2 If $\epsilon>0$ is sufficiently small, then there exists a solution $\left(x_{\epsilon}, r_{\epsilon}\right)$ of (12) such that

$$
\begin{gathered}
x_{\epsilon}=x_{0}+\tilde{x}_{\epsilon}, \quad r_{\epsilon}=r_{0}+\tilde{r}_{\epsilon} \quad \text { with } \tilde{x}_{\epsilon}, \tilde{r}_{\epsilon} \in H^{2} \cap C^{2}, \\
\left(\tilde{x}_{\epsilon}, \tilde{r}_{\epsilon}\right) \perp\left(x_{0}^{\prime}, r_{0}^{\prime}\right) \quad\left(\text { in } L^{2} \times L^{2}\right),
\end{gathered}
$$

and

where

$$
\left\|\left(\tilde{x}_{\epsilon}, \tilde{r}_{\epsilon}\right)\right\|_{1, \epsilon}=O\left(\epsilon^{2}\right)
$$

$$
\|(x, r)\|_{1, \epsilon}^{2}=\epsilon^{2}\left\|x^{\prime}\right\|_{L^{2}}^{2}+\left\|r^{\prime}\right\|_{L^{2}}^{2}+\|x\|_{L^{2}}^{2}+\|r\|_{L^{2}}^{2} .
$$

\section{The stability of the spectrum of the heteroclinic}

Theorem $\mathrm{B}$ is embodied in the corollary below. We consider a family of functions $\left(x_{\epsilon}(s), r_{\epsilon}(s)\right) \in$ $C^{2} \times C^{2}, \epsilon \geqslant 0$, satisfying

(H1) $\left(x_{\epsilon}(s), r_{\epsilon}(s)\right)$ are continuous in $\epsilon$ uniformly for $s \in \mathbb{R}$ with $\left(x_{0}(s), r_{0}(s)\right)$ as in Sec.2.2, $\lim _{|s| \rightarrow \infty} x_{\epsilon}, \lim _{|s| \rightarrow \infty} r_{\epsilon}$ exist, and $\left\|x_{\epsilon}^{\prime}\right\|_{\infty},\left\|r_{\epsilon}^{\prime}\right\|_{\infty},\left\|x_{\epsilon}^{\prime \prime}\right\|_{L^{2}},\left\|r_{\epsilon}^{\prime \prime}\right\|_{L^{2}}$ are bounded uniformly in $\epsilon$.

In this section we assume that $g_{x x}, g_{x r}, g_{r r}$ are evaluated at $\left(x_{\epsilon}, r_{\epsilon}\right)$ satisfying (H1). We define (using the same notation) the operator $L_{\epsilon}$ as in (27) but now with $\left(x_{\epsilon}, r_{\epsilon}\right)$ in place of $\left(x_{0}, r_{0}\right)$. From the asymptotic behavior of $g_{x x}, g_{x r}, g_{r r}$ as $|s| \rightarrow \infty$ it follows as in Sec. 2.3 that for small $\epsilon>0$ we have $\sigma_{\text {ess }}\left(L_{\epsilon}\right) \subset\left[C_{3} / 2, \infty\right)$.

We now consider the eigenvalue problem $L_{\epsilon} h=\lambda h, h=\left(h_{1}, h_{2}\right),\|h\|_{L^{2} \times L^{2}}=1$. We can perform steps 39 - 54 without any change ${ }^{1}$ However, in order to continue, we need the following lemma whose proof is an immediate consequence of (H1), Th. 3.1, p. 482 in [7], and [26).

LEMMA 7 Set $q(s, \epsilon)=g_{r r}-g_{x r}^{2} / g_{x x}$ and define the operator $B(\epsilon)$ in $L^{2}$ via

$$
B(\epsilon) h=-h^{\prime \prime}+q(s, \epsilon) h, \quad \text { with } D(B(\epsilon))=H^{2} .
$$

Then if $\epsilon \geqslant 0$ is sufficiently small, the essential spectrum of $B(\epsilon)$ is contained in $\left[C_{1} / 2, \infty\right)$ and the smallest eigenvalue $\mu_{1}(\epsilon)$ of $B(\epsilon)$ is simple, $\mu_{1}(\epsilon) \rightarrow 0$ and the corresponding $L^{2}$ normalized eigenfunction $y_{\epsilon}$ satisfies $y_{\epsilon} \rightarrow y$ in $H^{2}$ ( $y$ as in Sec. 2.6.

Using this, we can prove the analogs of the results in Secs. 2.2, 2.7, 2.8 and obtain (using the same notation):

THEOREM 3 If $\epsilon>0$ is sufficiently small and $\left(x_{\epsilon}, r_{\epsilon}\right)$ satisfies (H1), then the spectrum of $L_{\epsilon}$ has the following form: At the bottom of the spectrum, there is a simple eigenvalue $\lambda_{1}(\epsilon)$ with $\lambda_{1}(\epsilon)=O\left(\epsilon^{2}+\mu_{1}(\epsilon)\right)$. Furthermore, the normalized eigenfunction $\left(h_{1}^{\epsilon}, h_{2}^{\epsilon}\right)$ corresponding to $\lambda_{1}(\epsilon)$ satisfies

$$
\begin{gathered}
h_{2}^{\epsilon}=a_{\epsilon} y_{\epsilon}+p_{\epsilon}, \quad p_{\epsilon} \perp y_{\epsilon} \quad\left(\text { in } L^{2}\right), \\
a_{\epsilon} \in \mathbb{R}, \quad p_{\epsilon} \in H^{2}, \quad\left|a_{\epsilon}\right| \geqslant a_{0}, \quad\left\|p_{\epsilon}\right\|_{H^{2}}=O\left(\epsilon^{2}+\mu_{1}(\epsilon)\right)
\end{gathered}
$$

$\left(a_{0}, \lambda_{0}\right.$ are positive constants independent of $\epsilon$, but depending on the family $\left(x_{\epsilon}, r_{\epsilon}\right)$ ).

1 Except now instead of the boundedness of the derivatives of $g_{x r}, g_{x x}$, we use (H1). 
The following corollary, which completes the proofs of the theorems stated in the introduction, implies the stability of the spectrum of the heteroclinic orbit of (12) obtained in Sec.3

COROLlary 1 Let $\left(x_{\epsilon}, r_{\epsilon}\right)$ be the heteroclinic orbit of (12) given in Theorem 2 above. Then if $\epsilon>0$ is sufficiently small, the spectrum of the linearized operator $L_{\epsilon}$ about $\left(x_{\epsilon}, r_{\epsilon}\right)$ has the following form: zero is a simple eigenvalue at the bottom of the spectrum and the rest of the spectrum is contained in $\left[C_{29}, \infty\right)$ where $C_{29}>0$ (and as always with constants, independent of $\epsilon$ ).

Proof. It is easy to show that $\left(x_{\epsilon}, r_{\epsilon}\right)$ satisfies (H1) via 711 and that zero is an eigenvalue of $L_{\epsilon}$ with normalized eigenfunction $u_{\epsilon}^{\prime} /\left\|u_{\epsilon}^{\prime}\right\|$, where $u_{\epsilon}=\left(x_{\epsilon}, r_{\epsilon}\right)$. Now the corollary follows by Theorem 3 .

\section{REFERENCES}

1. Alikakos, N. D., Bates, P. W., Cahn, J. W., Fife, P. C., Fusco, G., \& Tanoglu, G. B. Analysis of a corner layer problem in anisotropic interfaces. Discrete Contin. Dyn. Syst. 6 (2005), 237-255. MR 2176291

2. Alikakos, N. D., Bates, P. W., \& Fusco, G. Slow motion for the Cahn-Hilliard equation in one space dimension. J. Differential Equations 90 (1991), 81-135. Zbl 0753.35042 MR 1094451

3. Alikakos, N. D., Fusco, G., \& KowalczyK, M. Finite-dimensional dynamics and interfaces intersecting the boundary: equilibria and quasi-invariant manifolds. Indiana Univ. Math. J. 45 (1996), 1119-1155. Zbl 0893.35051 MR 1444480

4. ARnol'D, V. I. Ordinary Differential Equations. Springer (1999).

5. Braun, R. J. Cahn, J. W., McFadden, G. B., \& Wheeler, A. A. Trans. Roy. Soc. London A 355 (1997), 1787.

6. CAHn, J. W., \& KiKUCHI, R. Theory of domain walls in ordered structures-I. Properties of absolute zero. J. Phys. Chem. Solids 20 (1961), 94-109.

7. Chow, S. N., \& HAle, J. K. Methods of Bifurcation Theory. Springer (1982). Zbl 0487.47039 MR 0660633

8. Fenichel, N. Geometric singular perturbation theory for ordinary differential equations. J. Differential Equations 31 (1979), 53-98. Zbl 0476.34034 MR 0524817

9. FIFE, P. Profile in the direction $(1,0,0)$. Manuscript.

10. Friedman, A. Partial Differential Equations. Holt, Rinehart and Winston (1969). Zbl 0224.35002 MR 0445088

11. Hale, J. K., \& KoçAK, H. Dynamics and Bifurcations. Springer (1991). Zbl 0745.58002 MR 1138981

12. Henry, D. Geometric Theory of Semilinear Parabolic Equations. Lecture Notes in Math. 840, Springer (1981). Zbl 0456.35001 MR 0610244

13. Hislop, P. D., \& SigaL, I. M. Introduction to Spectral Theory with Applications to Schrödinger Operators. Springer (1996). Z Zbl 0855.47002 MR 1361167

14. Jones, C. K. R. T. Geometric singular perturbation theory. L. Arnold, C. Jones, K. Mischaikow and G. Raugel, Dynamical Systems, Lecture Notes in Math. 1609, Springer (1995), 44-118. Zbl 0840.58040 MR 1374108

15. Landau, L. D., \& Lifshitz, E. M. Statistical Physics. 3rd ed., Part 1, Butterworth-Heinemann (1997).

16. McFadden, G. B., HAn, S. C., \& W. CAHn, J. Interface anisotopy in order-disorder transition on an HCP binary alloy. Preprint.

17. Nishiura, Y. Far-from-Equilibrium Dynamics. Transl. Math. Monogr. 209, Amer. Math. Soc. (1999). Zbl 1013.37001 MR 1903642 
18. Novick-CoHen, A., \& CAHN, J. W. Evolution equations for phase separation and ordering in binary alloys. J. Statist. Phys. 76 (1994), 877-909. Zbl 0840.35110

19. Porter, D. A., \& Easterling, K. E. Phase Transformations in Metals and Alloys. 2nd ed., Chapman and Hall (1992).

20. ReEd, M., \& Simon, B. Methods of Modern Mathematical Physics IV. Academic Press, New York (1978). Zbl 0401.47001 MR 0493421

21. Szmolyan, P. Analysis of a singularly perturbed traveling wave problem. SIAM J. Appl. Math. 52 (1992), 485-492. Zbl 0749.34026 MR 1154784

22. Tanoglu, G. B. Ph.D. Thesis, Univ. of Delaware, Dept. Appl. Math. (2000). 